\title{
Diffusiophoresis and electrophoresis of a charged sphere perpendicular to two plane walls
}

\author{
Yu C. Chang, Huan J. Keh* \\ Department of Chemical Engineering, National Taiwan University, Taipei 10617, Taiwan, Republic of China \\ Received 24 January 2008; accepted 4 March 2008 \\ Available online 16 April 2008
}

\begin{abstract}
The problem of diffusiophoretic and electrophoretic motions of a dielectric spherical particle in an electrolyte solution situated at an arbitrary position between two infinite parallel plane walls is studied theoretically in the quasisteady limit of negligible Peclet and Reynolds numbers. The applied electrolyte concentration gradient or electric field is uniform and perpendicular to the plane walls. The electric double layer at the particle surface is assumed to be thin relative to the particle radius and to the particle-wall gap widths, but the polarization effect of the diffuse ions in the double layer is incorporated. To solve the conservative equations, the general solution is constructed from the fundamental solutions in both cylindrical and spherical coordinates. The boundary conditions are enforced first at the plane walls by the Hankel transforms and then on the particle surface by a collocation technique. Numerical results for the diffusiophoretic and electrophoretic velocities of the particle relative to those of a particle under identical conditions in an unbounded solution are presented for various cases. The collocation results agree well with the approximate analytical solutions obtained by using a method of reflections. The presence of the walls can reduce or enhance the particle velocity, depending on the properties of the particle-solution system and the relative particle-wall separation distances. The boundary effects on diffusiophoresis and electrophoresis of a particle normal to two plane walls are found to be quite significant and complicated, and generally stronger than those parallel to the confining walls.
\end{abstract}

(c) 2008 Elsevier Inc. All rights reserved.

Keywords: Diffusiophoresis; Electrophoresis; Boundary effects; Thin but polarized double layer

\section{Introduction}

The electrokinetic transport phenomena of charged colloidal particles suspended in electrolyte solutions are of much fundamental and practical interest in the fields of chemical, biological, and electronic engineering and science. Electrophoresis, which results from the interaction between an applied electric field and the electric double layer surrounding a charged particle, is the most familiar example of various electrokinetic transport phenomena. The electrophoretic velocity $\mathbf{U}^{(0)}$ of a uniformly charged particle in an unbounded ionic solution is simply related to the uniformly imposed electric field $\mathbf{E}^{\infty}$ by the Smoluchowski equation [1-4],

$\mathbf{U}^{(0)}=\frac{\varepsilon \zeta}{\eta} \mathbf{E}^{\infty}$.

\footnotetext{
* Corresponding author.

E-mail address: huan@ccms.ntu.edu.tw (H.J. Keh).
}

In this equation, $\varepsilon$ and $\eta$ are the dielectric permittivity and viscosity, respectively, of the fluid, and $\zeta$ is the zeta potential at the particle surface.

Diffusiophoresis, which is the movement of a particle in response to the macroscopic concentration gradient of a solute, can be another example of electrokinetic motions. In an unbounded solution of a symmetrically charged electrolyte with a constant concentration gradient $\nabla n^{\infty}$, the diffusiophoretic velocity of a dielectric particle is [5-7]

$$
\mathbf{U}^{(0)}=\frac{\varepsilon \zeta}{\eta} \frac{k T}{Z e} \frac{\nabla n^{\infty}}{n^{\infty}(\mathbf{0})}\left(\alpha+\frac{\ln \cosh \bar{\zeta}}{\bar{\zeta}}\right)
$$

with the dimensionless parameters

$$
\begin{aligned}
& \alpha=\frac{\bar{D}_{2}-\bar{D}_{1}}{\bar{D}_{2}+\bar{D}_{1}}, \\
& \bar{\zeta}=\frac{Z e \zeta}{4 k T} .
\end{aligned}
$$


Here $n^{\infty}(\mathbf{0})$ is the macroscopic electrolyte concentration measured at the particle center $\mathbf{0}$ in the absence of the particle, $\bar{D}_{1}$ and $\bar{D}_{2}$ are the diffusion coefficients of the anion and cation, respectively, $Z$ is the absolute value of valences of ions, $e$ is the charge of a proton, $k$ is the Boltzmann constant, and $T$ is the absolute temperature.

Diffusiophoresis of a charged particle in an electrolyte solution can be considered as a linear combination of two effects: chemiphoresis due to the nonuniform adsorption of counterions and depletion of co-ions over the surface of the particle and electrophoresis driven by the macroscopic electric field generated by the electrolyte concentration gradient. For the special case of $\bar{D}_{2}=\bar{D}_{1}$ or $\alpha=0$, the induced macroscopic electric field disappears and Eq. (2) predicts that the particle movement (due to chemiphoresis only) is in the direction of increasing electrolyte concentration regardless of the sign of $\zeta$ and the particle velocity increases monotonically with an increase in the magnitude of $\zeta$.

As shown in Eqs. (1) and (2), the electrophoretic and diffusiophoretic velocities of a dielectric particle having a uniform zeta potential on its surface are independent of the particle size and shape. However, their validity is based on the assumptions that the local radii of curvature of the particle are much larger than the thickness of the electric double layer surrounding the particle surface and that the effect of polarization (relaxation effect) of the diffuse ions in the double layer due to nonuniform "osmotic" flow is negligible. In the past decades, important advances have been made in the evaluation of the electrophoretic and diffusiophoretic velocities of dielectric particles relaxing these assumptions.

O'Brien and White [8] took the double-layer distortion from equilibrium as a perturbation to obtain numerical solutions for the electrophoretic velocity of a spherical particle of radius $a$ in a $\mathrm{KCl}$ solution which were applicable to arbitrary values of $\zeta$ and $\kappa a$, where $\kappa^{-1}$ is the Debye screening length equal to $\left(\varepsilon k T / 2 Z^{2} e^{2} n^{\infty}\right)^{1 / 2}$. On the other hand, Dukhin and Derjaguin [3] obtained an analytical expression for the electrophoretic mobility of a dielectric sphere surrounded by a thin but polarized double layer in the solution of a symmetric electrolyte. Later, O'Brien [9] generalized this analysis to the case of electrophoretic motion of a charged sphere in the solution containing an arbitrary combination of electrolytes. The essence of this thin-layer polarization approach is that a thin diffuse layer can still transport a significant amount of electrolyte ions so as to affect the ionic transport outside the diffuse layer. The result for the electrophoretic velocity of a dielectric sphere with a thin but polarized double layer in a symmetric electrolyte solution can be expressed as [10]

$\mathbf{U}_{0}=\frac{\varepsilon \zeta}{3 \eta} \mathbf{E}^{\infty}\left[2+c_{1}+c_{2}+\left(c_{1}-c_{2}\right) \frac{\ln \cosh \bar{\zeta}}{\bar{\zeta}}\right]$,

where coefficients $c_{1}$ and $c_{2}$ are defined by Eqs. (A.6a) and (A.6b). A comparison of Eq. (5) with the numerical results for the $\mathrm{KCl}$ solution [8] shows that the thin-layer polarization model is quite good over a wide range of zeta potentials when $\kappa a>20$. If $|\zeta|$ is small and $\kappa a$ is large, the interaction between the diffuse counterions and the particle surface is weak and the polarization of the double layer is also weak. In the limit of

$\mathrm{e}^{2|\bar{\zeta}|} / \kappa a \rightarrow 0$,

$c_{1}=c_{2}=1 / 2$ and Eq. (5) reduces to the Smoluchowski equation (1). Unlike the prediction of Eq. (1), the electrophoretic velocity given by Eq. (5) in general is not a monotonic function of $\bar{\zeta}$ for a finite value of $\kappa a$.

On the other hand, Prieve and Roman [11] obtained numerical solutions of the diffusiophoretic velocity over a broad range of $\bar{\zeta}$ and $\kappa a$ for a charged sphere in concentration gradients of symmetric electrolytes $(\mathrm{KCl}$ or $\mathrm{NaCl})$ using the method of O'Brien and White [8]. Analytical expressions for the velocity of a dielectric sphere with a thin but polarized double layer undergoing diffusiophoresis in ionic solutions have also been derived $[12,13]$, and the result for this diffusiophoretic velocity in a symmetric electrolyte solution is

$$
\begin{aligned}
\mathbf{U}_{0}= & \frac{\varepsilon \zeta}{3 \eta} \frac{k T}{Z e} \frac{\nabla n^{\infty}}{n^{\infty}(\mathbf{0})}\left\{c_{1}^{\prime}-c_{2}^{\prime}+\alpha\left(2+c_{1}+c_{2}\right)\right. \\
& \left.+\left[2+c_{1}^{\prime}+c_{2}^{\prime}+\alpha\left(c_{1}-c_{2}\right)\right] \frac{\ln \cosh \bar{\zeta}}{\bar{\zeta}}\right\},
\end{aligned}
$$

where coefficients $c_{1}^{\prime}$ and $c_{2}^{\prime}$ are defined by Eqs. (A.6c) and (A.6d). When $\kappa a>20$, the agreement between Eq. (7) and the numerical solutions [11] is excellent for all reasonable values of the zeta potential. In the limiting situation given by Eq. (6), the effect of double-layer polarization disappears, $c_{1}=c_{2}=c_{1}^{\prime}=$ $c_{2}^{\prime}=1 / 2$, and Eq. (7) reduces to Eq. (2). Even for the case of $\bar{D}_{2}=\bar{D}_{1}$, the particle velocity given by Eq. (7) for a finite value of $\kappa a$ may not be a monotonic function of the magnitude of $\bar{\zeta}$ and its direction can reverse (toward lower electrolyte concentration) when $|\bar{\zeta}|$ becomes large.

An examination of Eqs. (5) and (7) indicates that the effect of polarization of the diffuse layer is to decrease the particle velocity. The reason for this outcome is that the transport of the diffuse ions within the double layer reduces the local electrolyte gradient or electric field along the particle surface. Numerical calculations of Eqs. (5) and (7) show that, even when $\kappa a$ is as large as 300 , the effect of ionic transport inside the diffuse layer cannot be ignored if $|\zeta|$ equals several $k T / e$.

In real situations of electrophoresis and diffusiophoresis, particles are not isolated and the surrounding fluid is externally bounded by solid walls [14-17]. Thus, it is important to determine if the presence of neighboring boundaries significantly affects the movement of particles. In the limiting case that Eqs. (1) and (2) are applicable, the normalized velocity field of the immense fluid that is dragged by a particle during diffusiophoresis is the same as for electrophoresis of the particle [8]; thus, the boundary effects on electrophoresis under the situation of infinitesimally thin double layer (satisfying Eq. (6)), which have been studied extensively in the past [17-24], can be taken to interpret those in diffusiophoresis.

When the polarization effect of diffuse ions in the electric double layer surrounding the particle is considered, the boundary effects on diffusiophoresis can be quite different from those on electrophoresis. Through the use of a boundary collocation technique, the diffusiophoretic and electrophoretic motions of a 


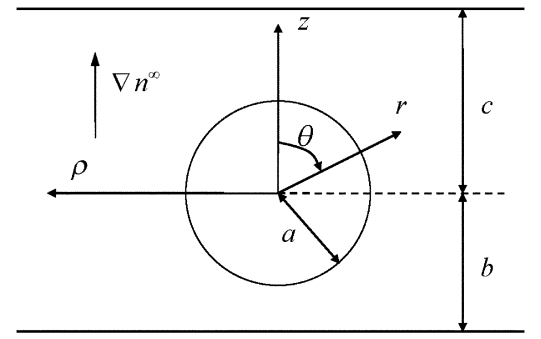

Fig. 1. Geometrical sketch for the diffusiophoretic motion of a spherical particle perpendicular to two plane walls at an arbitrary position between them.

colloidal sphere with a thin but polarized diffuse layer perpendicular to a plane wall have been examined [25]. The wall effect in each transport mechanism was found to be a complicated function of the properties of the particle and ions. Recently, the diffusiophoretic and electrophoretic motions of a colloidal sphere with a thin polarized diffuse layer parallel to two plane walls at an arbitrary position between them were also investigated by Chen and Keh [26] using the boundary collocation method. Numerical results of the wall correction to Eqs. (5) and (7) for the particle mobilities were presented for various values of the relative separation distances and other relevant parameters.

This paper is an extension of the previous works $[25,26]$ to the situation of the diffusiophoretic and electrophoretic motions of a dielectric sphere with a thin but polarized double layer perpendicular to two plane walls at an arbitrary position between them. The quasisteady equations of conservation applicable to each system are solved by using both the boundary-collocation technique numerically and a method of reflections analytically. The numerical solutions of the particle velocities are obtained with good convergence for various cases and agree well with the approximate analytical solutions. For the case of motions normal to a single plane wall, our results are in excellent agreement with those available in the literature $[18,25]$. Because the governing equations and boundary conditions concerning the general problem of diffusiophoresis and electrophoresis of a particle at an arbitrary position between two parallel plane walls in an arbitrary direction are linear, its solution can be obtained as a superposition of the solutions for its two subproblems: motion parallel to the plane walls, which was previously examined [26], and motion normal to the confining walls, which is considered in this paper.

\section{Analysis for diffusiophoresis}

We consider, in this section, the quasisteady diffusiophoretic motion of a dielectric spherical particle of radius $a$ in a solution of a symmetrically charged electrolyte perpendicular to two infinite plane walls whose distances from the center of the particle are $b$ and $c$, as shown in Fig. 1. Here $(\rho, \phi, z)$ and $(r, \theta, \phi)$ denote the circular cylindrical and spherical coordinate systems, respectively, and the origin of coordinates is chosen at the particle center. A linear electrolyte concentration field $n^{\infty}(z)$ with a uniform gradient in the $z$ direction is imposed in the ambient fluid far removed from the particle. The particle is charged uniformly on the surface, and the thickness of the electric double layers surrounding the particle and adjacent to the plane walls is assumed to be small in comparison with the radius of the particle and the spacing between the particle and each plane wall [i.e., $\kappa a \gg 1, \kappa(b-a) \gg 1$, and $\kappa(c-a) \gg 1$, which is valid for most practical situations where the electrolyte solution has a relatively strong ionic strength]. Hence, the fluid phase can be divided into two regions: an "inner" region defined as the thin double layer adjacent to each solid surface and an "outer" region defined as the remainder of the fluid, which is electrically neutral. The purpose is to obtain the correction to Eq. (7) for the particle velocity due to the presence of the plane walls.

To determine the diffusiophoretic velocity of the particle, it is necessary to ascertain the electrochemical potential and velocity fields in the fluid phase.

\subsection{Electrochemical potential distribution}

The diffusiophoretic motion of a particle can be considered quasisteady if the Peclet and Reynolds numbers of the system are small. Hence, the conservation of each ionic species for the outer region of the fluid solution is governed by $[9,12]$

$\nabla^{2} \mu_{m}=0, \quad m=1,2$.

In the above Laplace equation, $\mu_{m}$ is the electrochemical potential energy of ionic species $m$ defined by

$\mu_{m}=\mu_{m}^{0}+k T \ln n_{m}+z_{m} e \Phi$,

where $\mu_{m}^{0}$ is a constant, $n_{m}$ and $z_{m}$ are the concentration and valence, respectively, of type $m$ ions, and $\Phi$ is the electric potential. $m$ equal to 1 and 2 refers to the anion and cation, respectively, so $-z_{1}=z_{2}=Z>0$. Note that, in the outer region, $n_{1}=n_{2}=n$, and both $n$ and $\Phi$ also satisfy Laplace's equation.

The boundary condition at the particle "surface" (outer limit of the thin double layer) can be obtained by solving for the electrochemical potentials in the inner region and using a matching procedure to ensure a continuous solution in the whole fluid phase, with the result $[9,12]$

$$
\begin{gathered}
r=a^{+}: \quad \frac{\partial \mu_{m}}{\partial r}=-\sum_{i=1}^{2} \beta_{m i} \frac{1}{r^{2} \sin \theta} \frac{\partial}{\partial \theta}\left(\sin \theta \frac{\partial \mu_{m}}{\partial \theta}\right), \\
m=1,2,
\end{gathered}
$$

where the relaxation coefficients

$$
\begin{aligned}
\beta_{11}= & \frac{1}{\kappa}\left[4\left(1+\frac{3 f_{1}}{Z^{2}}\right) \mathrm{e}^{\bar{\zeta}} \sinh \bar{\zeta}-\frac{12 f_{1}}{Z^{2}}(\bar{\zeta}+\ln \cosh \bar{\zeta})\right], \\
\beta_{12}= & -\frac{1}{\kappa}\left(\frac{12 f_{1}}{Z^{2}}\right) \ln \cosh \bar{\zeta}, \\
\beta_{21}= & -\frac{1}{\kappa}\left(\frac{12 f_{2}}{Z^{2}}\right) \ln \cosh \bar{\zeta}, \\
\beta_{22}= & \frac{1}{\kappa}\left[-4\left(1+\frac{3 f_{2}}{Z^{2}}\right) \mathrm{e}^{-\bar{\zeta}} \sinh \bar{\zeta}\right. \\
& \left.+\frac{12 f_{2}}{Z^{2}}(\bar{\zeta}-\ln \cosh \bar{\zeta})\right] .
\end{aligned}
$$


In the above equation, $f_{m}=2 \varepsilon k^{2} T^{2} / 3 \eta e^{2} \bar{D}_{m}$ and $\bar{\zeta}$ was defined by Eq. (4). To obtain Eqs. (10) and (11), it was assumed that the concentration of each ionic species within the electric double layer is related to the electric potential energy by a Boltzmann distribution. Evidently, in the limit of Eq. (6), $\beta_{11}=\beta_{12}=\beta_{21}=\beta_{22}=0$.

Since the ionic electrochemical potentials far away from the particle approach the undisturbed distributions, we can write

$z=c,-b: \quad \mu_{m}=\mu_{m \infty}$,

$\rho \rightarrow \infty,-b<z<c: \quad \mu_{m} \rightarrow \mu_{m_{\infty}}$,

where

$\mu_{m \infty}=\mu_{m}^{0}+k T\left[1-(-1)^{m} \alpha\right] \ln n^{\infty}$

and $\alpha$ was defined by Eq. (3). The second term in the brackets of Eq. (14) represents the contribution from the macroscopic electric field induced by the difference between cation and anion diffusion rates [6]. Note that the ionic electrochemical potentials have been set equal to different constants at the two parallel plane walls (which must be electrically conducting ones) to allow a uniform electrolyte concentration gradient in their normal direction far from the particle. This situation can be generated experimentally, as an example, from employing two planar electrodes on which some electrochemical reactions occur.

The electrochemical potential distributions, which are governed by the linear Laplace equation, can be expressed as the superposition

$\mu_{m}=\mu_{m \infty}+\mu_{m \mathrm{w}}+\mu_{m \mathrm{p}}, \quad m=1,2$.

Here $\mu_{m \text { w }}$ is a separable solution of Eq. (8) in cylindrical coordinates that represents the disturbance produced by the plane walls and is given by a Fourier-Bessel integral,

$\mu_{m \mathrm{w}}=\int_{0}^{\infty}\left[X(\omega) \mathrm{e}^{\omega z}+Y(\omega) \mathrm{e}^{-\omega z}\right] \omega J_{0}(\omega \rho) d \omega$,

where $J_{n}$ is the Bessel function of the first kind of order $n$, and $X(\omega)$ and $Y(\omega)$ are unknown functions of the separation variable $\omega$. The last term on the right-hand side of Eq. (15), $\mu_{m \mathrm{p}}$, is a separable solution of Eq. (8) in spherical coordinates representing the disturbance generated by the particle and is given by an infinite series in harmonics,

$\mu_{m \mathrm{p}}=\sum_{n=0}^{\infty} R_{m n} r^{-n-1} P_{n}(\cos \theta)$,

where $P_{n}$ is the Legendre polynomial of order $n$ and $R_{m n}$ are unknown constants. Note that a solution for $\mu_{m}$ of the form given by Eqs. (15)-(17) immediately satisfies the boundary condition at infinity in Eq. (13).

Substituting the electrochemical potential distribution $\mu_{m}$ given by Eqs. (15)-(17) into the boundary condition (12) and applying the Hankel transform on the variable $\rho$ lead to a solution for the functions $X(\omega)$ and $Y(\omega)$ in terms of the coefficients $R_{m n}$. After the substitution of this solution into Eqs. (15)-(17), $\mu_{m}$ can be expressed as $\mu_{m}=\mu_{m \infty}+\sum_{n=0}^{\infty} R_{m n} \delta_{n}^{(1)}(r, \theta), \quad m=1,2$,

where the function $\delta_{n}^{(1)}(r, \theta)$ is defined by Eq. (B.1) in Appendix $\mathrm{B}$ (in which the definite integral must be performed numerically).

Application of the boundary condition given by Eq. (10) to Eq. (18) yields

$$
\begin{aligned}
\sum_{n=0}^{\infty} \sum_{m=1}^{2} R_{m n} R_{m}^{\prime}(\theta)= & k T \frac{\left|\nabla n^{\infty}\right|}{n^{\infty}(\mathbf{0})}\left[\left(1-\frac{2 \beta_{11}}{a}\right)(1+\alpha)\right. \\
& \left.-\frac{2 \beta_{12}}{a}(1-\alpha)\right] \cos \theta, \\
\sum_{n=0}^{\infty} \sum_{m=1}^{2} R_{m n} R_{m}^{\prime \prime}(\theta)= & k T \frac{\left|\nabla n^{\infty}\right|}{n^{\infty}(\mathbf{0})}\left[\left(1-\frac{2 \beta_{22}}{a}\right)(1-\alpha)\right. \\
& \left.-\frac{2 \beta_{21}}{a}(1+\alpha)\right] \cos \theta,
\end{aligned}
$$

where

$R_{1}^{\prime}(\theta)=\left(\frac{2 \beta_{11}}{a}-1\right) \delta_{n}^{(2)}(a, \theta)+\beta_{11} \delta_{n}^{(4)}(a, \theta)$,

$R_{2}^{\prime}(\theta)=\beta_{12}\left[\frac{2}{a} \delta_{n}^{(2)}(a, \theta)+\delta_{n}^{(4)}(a, \theta)\right]$,

$R_{1}^{\prime \prime}(\theta)=\beta_{21}\left[\frac{2}{a} \delta_{n}^{(2)}(a, \theta)+\delta_{n}^{(4)}(a, \theta)\right]$,

$R_{2}^{\prime \prime}(\theta)=\left(\frac{2 \beta_{22}}{a}-1\right) \delta_{n}^{(2)}(a, \theta)+\beta_{22} \delta_{n}^{(4)}(a, \theta)$,

and the definitions of functions $\delta_{n}^{(2)}(r, \theta)$ and $\delta_{n}^{(4)}(r, \theta)$ are given by Eqs. (B.2) and (B.4) (in which the integration must be performed numerically).

To satisfy the condition (19) exactly along the entire surface of the particle would require the solution of the entire infinite array of unknown constants $R_{m n}$. However, the collocation method [25-27] enforces the boundary condition at a finite number of discrete points on any semicircular longitudinal generating arc of the sphere (from $\theta=0$ to $\pi$ ) and truncates the infinite series in Eq. (18) into a finite one. If the spherical boundary is approximated by satisfying the condition (10) at $M$ discrete points on its generating arc, the infinite series in Eq. (18) is truncated after $M$ terms, resulting in a system of $2 M$ simultaneous linear algebraic equations in the truncated form of Eq. (19). This matrix equation can be numerically solved to yield the $2 M$ unknown constants $R_{1 n}$ and $R_{2 n}$ required in the truncated form of Eq. (18) for the electrochemical potential distributions. The accuracy of the boundary-collocation/truncation technique can be improved to any degree by taking a sufficiently large value of $M$. Naturally, as $M \rightarrow \infty$ the truncation error vanishes and the overall accuracy of the solution depends only on the numerical integration required in evaluating the functions $\delta_{n}^{(2)}(r, \theta)$ and $\delta_{n}^{(4)}(r, \theta)$ in Eq. (20). 


\subsection{Fluid velocity distribution}

Having obtained the solution for the ionic electrochemical potential distributions on the particle surface that drive the diffusiophoretic migration, we can now proceed to find the flow field. The fluid solution is assumed to be incompressible and Newtonian. Owing to the low Reynolds number encountered in diffusiophoresis, the fluid motion in the outer region is governed by the quasisteady fourth-order differential equation for viscous axisymmetric creeping flows,

$E^{2}\left(E^{2} \Psi\right)=0$,

in which the Stokes stream function $\Psi$ is related to the components of fluid velocity $\mathbf{v}$ in cylindrical coordinates by $\left(v_{\phi}=0\right)$

$v_{\rho}=\frac{1}{\rho} \frac{\partial \Psi}{\partial z}$,

$v_{z}=-\frac{1}{\rho} \frac{\partial \Psi}{\partial \rho}$

and the Stokes operator $E^{2}$ has the form

$E^{2}=\rho \frac{\partial}{\partial \rho}\left(\frac{1}{\rho} \frac{\partial}{\partial \rho}\right)+\frac{\partial^{2}}{\partial z^{2}}$.

The boundary conditions for the fluid velocity at the particle surface $[9,12]$, on the plane walls, and far from the particle are

$r=a^{+}: \quad \mathbf{v}=U \mathbf{e}_{z}+\sum_{m=1}^{2} W_{m} \frac{\partial \mu_{m}}{r \partial \theta} \mathbf{e}_{\theta}$,

$z=c,-b: \quad \mathbf{v}=\mathbf{0}$,

$\rho \rightarrow \infty: \quad \mathbf{v} \rightarrow \mathbf{0}$,

where $W_{m}$ are coefficients for the apparent slip velocity (across the thin electric double layer) at the particle surface due to the diffusioosmotic effect (a linear combination of both chemiosmotic and electroosmotic effects),

$W_{m}=\frac{2 \varepsilon}{\eta} \frac{k T}{(Z e)^{2}}\left[(-1)^{m} \bar{\zeta}-\ln \cosh \bar{\zeta}\right]$,

$\mathbf{e}_{z}$ and $\mathbf{e}_{\rho}$ are the unit vectors in cylindrical coordinates, $\mathbf{e}_{r}$ and $\mathbf{e}_{\theta}$ are the unit vectors in spherical coordinates, and $U$ is the diffusiophoretic velocity of the particle to be determined. Since the ionic electrochemical potentials are constants at the two conducting plane walls as set in Eq. (12), no apparent slip velocity occurs there as indicated by Eq. (25).

To solve the flow field, we express the stream function in the form

$\Psi=\Psi_{\mathrm{w}}+\Psi_{\mathrm{p}}$

Here $\Psi_{\mathrm{w}}$ is a Fourier-Bessel integral solution of Eq. (21) in cylindrical coordinates that represents the disturbance produced by the plane walls and is given by

$$
\begin{aligned}
\Psi_{\mathrm{w}}= & \int_{0}^{\infty}\left[A(\omega) \mathrm{e}^{\omega z}+B(\omega) \mathrm{e}^{-\omega z}+C(\omega) \omega z \mathrm{e}^{\omega z}\right. \\
& \left.+D(\omega) \omega z \mathrm{e}^{-\omega z}\right] \rho J_{1}(\omega \rho) d \omega,
\end{aligned}
$$

where $A(\omega), B(\omega), C(\omega)$, and $D(\omega)$ are unknown functions of $\omega$. The second part of $\Psi$, denoted by $\Psi_{\mathrm{p}}$, is a separable solution of Eq. (21) in spherical coordinates representing the disturbance generated by the charged sphere and is given by

$\Psi_{\mathrm{p}}=\sum_{n=2}^{\infty}\left(B_{n} r^{-n+1}+D_{n} r^{-n+3}\right) G_{n}^{-1 / 2}(\cos \theta)$,

where $G_{n}^{-1 / 2}$ is the Gegenbauer polynomial of the first kind of order $n$ and degree $-1 / 2 ; B_{n}$ and $D_{n}$ are unknown constants. Note that the boundary condition in Eq. (26) is immediately satisfied by a solution of the form given by Eqs. (28)-(30).

Substituting the stream function $\Psi$ given by Eqs. (28)(30) into the boundary conditions in Eq. (25) and applying the Hankel transform on the variable $\rho$ lead to a solution for $A(\omega), B(\omega), C(\omega)$, and $D(\omega)$ in terms of the coefficients $B_{n}$ and $D_{n}$. After the substitution of this solution into Eqs. (28) (30), the fluid velocity components can be expressed as

$v_{\rho}=\sum_{n=2}^{\infty}\left[B_{n} \gamma_{1 n}^{(1)}(r, \theta)+D_{n} \gamma_{2 n}^{(1)}(r, \theta)\right]$,

$v_{z}=\sum_{n=2}^{\infty}\left[B_{n} \gamma_{1 n}^{(2)}(r, \theta)+D_{n} \gamma_{2 n}^{(2)}(r, \theta)\right]$,

where the definitions of functions $\gamma_{i n}^{(j)}(r, \theta)$ for $i$ and $j$ equal to 1 or 2 are given by Eqs. (B.5) and (B.6) (in which the integration must be performed numerically).

The only boundary condition that remains to be satisfied is that on the particle surface. Substituting Eqs. (18) and (31) into Eq. (24), one obtains

$\sum_{n=2}^{\infty}\left[B_{n} \gamma_{1 n}^{(1)}(a, \theta)+D_{n} \gamma_{2 n}^{(1)}(a, \theta)\right]=\sum_{m=1}^{2} W_{m} F_{m}(\theta) \cos \theta$,

$\sum_{n=2}^{\infty}\left[B_{n} \gamma_{1 n}^{(2)}(a, \theta)+D_{n} \gamma_{2 n}^{(2)}(a, \theta)\right]=U-\sum_{m=1}^{2} W_{m} F_{m}(\theta) \sin \theta$,

where

$F_{m}(\theta)=-k T\left[1-(-1)^{m} \alpha\right] \frac{\left|\nabla n^{\infty}\right|}{n^{\infty}(\mathbf{0})} \sin \theta+\sum_{n=0}^{\infty} R_{m n} \delta_{n}^{(3)}(a, \theta)$

and the definition of the function $\delta_{n}^{(3)}(r, \theta)$ is given by Eq. (B.3). The first $2 M$ coefficients $R_{m n}$ have been determined through the procedure given in the previous subsection.

Equation (32) can be satisfied by utilizing the boundarycollocation technique presented for the solution of the electrochemical potential field. Along a longitudinal generating arc at the particle surface, Eq. (32) is applied at $N$ discrete points (values of $\theta$ between 0 and $\pi$ ) and the infinite series in Eq. (31) are truncated after $N$ terms. This generates a set of $2 N$ linear algebraic equations for the $2 N$ unknown coefficients $B_{n}$ and $D_{n}$. The fluid velocity field is completely obtained once these coefficients are solved for a sufficiently large number of $N$. 


\subsection{Derivation of the particle velocity}

The hydrodynamic force acting on the spherical particle can be determined from $[27,28]$

$F=4 \pi \eta D_{2}$.

This expression shows that only the lowest-order coefficient $D_{2}$ contributes to the drag force exerted on the particle by the fluid.

Since the particle is freely suspended in the surrounding fluid, the net force acting on the particle must vanish. Applying this constraint to Eq. (34), one has

$D_{2}=0$

To determine the diffusiophoretic velocity $U$ of the particle, Eq. (35) and the $2 N$ algebraic equations resulting from Eq. (32) are to be solved simultaneously.

\section{Results and discussion for diffusiophoresis}

The numerical results for the diffusiophoretic velocity of a charged spherical particle perpendicular to two plane walls at an arbitrary position between them, obtained by using the boundary-collocation method described in the previous section, are presented in this section. The system of linear algebraic equations to be solved for the coefficients $R_{1 n}$ and $R_{2 n}$ is constructed from Eq. (19), while that for $B_{n}$ and $D_{n}$ is composed of Eq. (32). All the numerical integrations to evaluate the functions $\delta_{n}^{(i)}$ and $\gamma_{i n}^{(j)}$ were done by the 180 -point Gauss-Laguerre guadrature.

When specifying the points along the half-circular generating arc of the spherical particle where the boundary conditions are to be exactly satisfied, the first points that should be chosen are $\theta=0$ and $\pi$, since these stagnation points control the gaps between the particle and the plane walls. In addition, the point $\theta=\pi / 2$ which defines the projected area of the particle normal to the direction of motion is also important. However, an examination of the systems of linear algebraic equations (19) and (32) shows that the matrix equations become singular if these points are used. To overcome this difficulty, these points are replaced by closely adjacent basic points, i.e., $\theta=\delta, \pi / 2-\delta, \pi / 2+\delta$, and $\pi-\delta$ [25-27]. Additional points along the generating arc are selected as mirror-image pairs about the equatorial plane $\theta=\pi / 2$ to divide the two quarter-circular arcs of the particle into equal segments. The optimum value of $\delta$ in this work is found to be $0.01^{\circ}$, with which the numerical results of the particle velocity for various values of the parameters $\alpha, f_{1}, Z$, $\zeta e / k T, \kappa a, b /(b+c)$, and $a / b$ converge satisfactorily.

\subsection{Motion normal to a single plane wall}

Some of the typical boundary-collocation solutions for the diffusiophoretic velocity of a dielectric sphere normal to a single plane wall (with $c \rightarrow \infty$ ) for different values of the parameters $\alpha, Z, \zeta e / k T$, and $a / b$ are presented in Table 1 . The diffusiophoretic velocity of an identical particle in an infinite fluid, $U_{0}$, given by Eq. (7), is used to normalize the wall-corrected

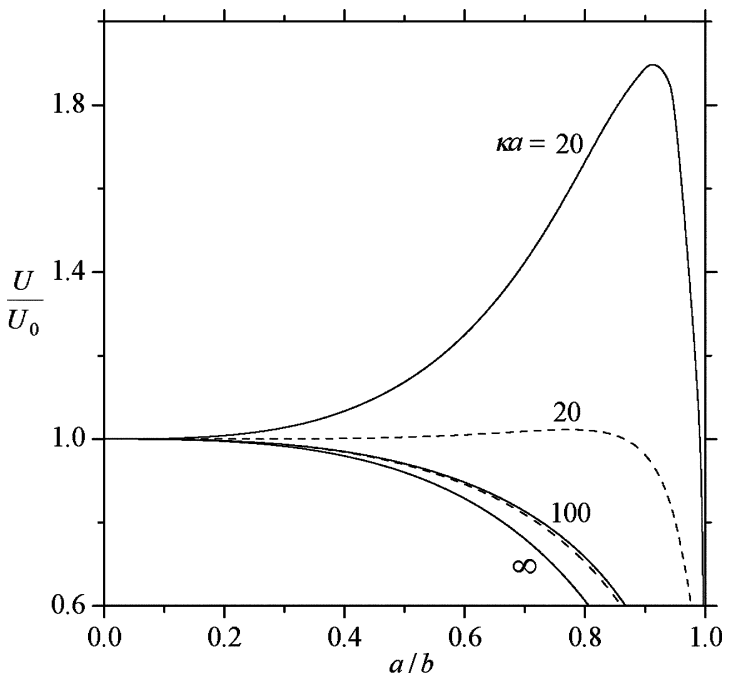

Fig. 2. Plots of the normalized diffusiophoretic velocity $U / U_{0}$ of a spherical particle perpendicular to a plane wall (with $c \rightarrow \infty$ ) versus the separation parameter $a / b$ with $Z=1, f_{1}=0.2$, and $\zeta e / k T=-5$ for various values of $\kappa a$. The solid curves represent the case of $\alpha=0$ and the dashed curves denote the case of $\alpha=-0.2$.

values. All of the results obtained under the collocation scheme converge satisfactorily to at least the significant figures shown in the table. The accuracy and convergence behavior of the truncation technique is principally a function of the ratio $a / b$. For the difficult case with $a / b=0.999$, the numbers of collocation points $M=N=200$ are sufficiently large to achieve this convergence. Our collocation results in Table 1 can be found to agree excellently with the existing numerical solutions obtained by using a similar boundary-collocation method [25]. Note that the electrolytes associated with $\alpha=0$ and -0.2 (taking $Z=1$ and $f_{1}=0.2$ ) in the table are very close to the aqueous solutions of $\mathrm{KCl}$ and $\mathrm{NaCl}$, respectively. As shown in Table 1, the particle migrates with the velocity that would exist in the absence of the wall, given by Eq. (7), as $a / b \rightarrow 0$. The diffusiophoretic velocity then may decrease or increase, or even reverse its direction, as the particle approaches the wall (with increasing $a / b$ ), depending on the combination of the relevant parameters. In any case, the particle velocity vanishes at the limit $a / b \rightarrow 1$.

In Appendix A, an approximate analytical solution for the same diffusiophoretic motion as that considered here is also obtained by using a method of reflections. The particle velocity is given by Eq. (A.28), which is a power series expansion in $\lambda(=a / b)$. The values of the wall-corrected normalized particle velocity calculated from this asymptotic solution, with the $O\left(\lambda^{9}\right)$ term neglected, are also listed in Table 1 for comparison. It can be seen that the asymptotic formula (A.28) from the method of reflections for the normalized particle mobility $U / U_{0}$ agrees quite well with the collocation results as long as $\lambda \leqslant 0.6$; the errors in most cases are less than $2 \%$. However, the accuracy of Eq. (A.28) deteriorates rapidly, as expected, when the relative spacing between the particle and the plane wall becomes small (say, $\lambda \geqslant 0.8$ ). The prediction of Eq. (A.28) may overestimate or underestimate the diffusiophoretic velocity of the particle, depending on the combination of the relevant parameters. 
Table 1

Normalized diffusiophoretic velocity of a dielectric sphere perpendicular to a single plane wall (with $c \rightarrow \infty$ ) obtained from the boundary-collocation method for the case $f_{1}=0.2$ and $\kappa a=100$

\begin{tabular}{|c|c|c|c|c|c|c|c|c|}
\hline \multirow[t]{3}{*}{$a / b$} & \multicolumn{8}{|l|}{$U / U_{0}$} \\
\hline & \multicolumn{4}{|l|}{$\alpha=0$} & \multicolumn{4}{|c|}{$\alpha=-0.2$} \\
\hline & $Z=1$ & & $Z=2$ & & $Z=1$ & & $Z=2$ & \\
\hline & $\zeta e / k T=$ & & & & & & & \\
\hline 0.2 & 0.99542 & $(0.99542)$ & 0.99575 & $(0.99575)$ & 0.99900 & (0.99899) & 0.99629 & $(0.99629)$ \\
\hline 0.4 & 0.96340 & $(0.96333)$ & 0.96616 & $(0.96604)$ & 0.99363 & $(0.99297)$ & 0.97066 & $(0.97047)$ \\
\hline 0.6 & 0.87088 & $(0.87069)$ & 0.88114 & $(0.87986)$ & 0.98667 & $(0.97092)$ & 0.89819 & $(0.89482)$ \\
\hline 0.8 & 0.65020 & $(0.66431)$ & 0.67774 & $(0.68129)$ & 0.97061 & $(0.83775)$ & 0.72431 & $(0.70793)$ \\
\hline 0.9 & 0.43157 & $(0.49515)$ & 0.46989 & $(0.50975)$ & 0.86353 & $(0.61297)$ & 0.53351 & $(0.52998)$ \\
\hline 0.95 & 0.26107 & $(0.38991)$ & 0.29872 & $(0.39853)$ & 0.64904 & $(0.41310)$ & 0.35809 & $(0.40651)$ \\
\hline 0.99 & 0.06415 & $(0.29505)$ & 0.08142 & $(0.29507)$ & 0.20075 & $(0.18983)$ & 0.10512 & $(0.28608)$ \\
\hline 0.995 & 0.03314 & & 0.04341 & & 0.10741 & & 0.05692 & \\
\hline \multirow[t]{2}{*}{0.999} & 0.0068 & & 0.0093 & & 0.0228 & & 0.0124 & \\
\hline & $\zeta e / k T=$ & & & & & & & \\
\hline 0.2 & 0.99628 & $(0.99628)$ & 1.00286 & (1.00286) & 0.99697 & $(0.99697)$ & 0.94469 & $(0.94471)$ \\
\hline 0.4 & 0.97053 & $(0.97037)$ & 1.02472 & (1.02412) & 0.97634 & $(0.97609)$ & 0.54463 & $(0.54846)$ \\
\hline 0.6 & 0.89716 & $(0.89450)$ & 1.09373 & (1.07872) & 0.91883 & $(0.91384)$ & -0.65639 & $(-0.54796)$ \\
\hline 0.8 & 0.72012 & $(0.70992)$ & 1.24760 & (1.10887) & 0.77856 & $(0.74581)$ & -3.45318 & $(-2.33078)$ \\
\hline 0.9 & 0.53023 & $(0.53827)$ & 1.32572 & (1.01832) & 0.61143 & $(0.56930)$ & -5.66735 & $(-2.95219)$ \\
\hline 0.95 & 0.36136 & $(0.42113)$ & 1.27559 & $(0.90452)$ & 0.44068 & $(0.43969)$ & -6.56694 & $(-2.91781)$ \\
\hline 0.99 & 0.11668 & $(0.30816)$ & 0.89325 & $(0.76140)$ & 0.15405 & $(0.30872)$ & -5.35464 & $(-2.58919)$ \\
\hline 0.995 & 0.06661 & & 0.71943 & & 0.08961 & & -4.41782 & \\
\hline 0.999 & 0.0164 & & 0.4096 & & 0.0226 & & -2.5812 & \\
\hline
\end{tabular}

Note. The figures in parentheses are asymptotic solutions calculated using Eq. (A.28).

Table 2

Normalized diffusiophoretic velocity of a dielectric sphere perpendicular to two equally distant plane walls (with $c=b$ ) obtained from the boundary-collocation method for the case $f_{1}=0.2$ and $\kappa a=100$

\begin{tabular}{|c|c|c|c|c|c|c|c|c|}
\hline \multirow[t]{3}{*}{$a / b$} & \multicolumn{8}{|l|}{$U / U_{0}$} \\
\hline & \multicolumn{4}{|l|}{$\alpha=0$} & \multicolumn{4}{|c|}{$\alpha=-0.2$} \\
\hline & $Z=1$ & & $Z=2$ & & $Z=1$ & & $Z=2$ & \\
\hline & $\zeta e / k T=$ & & & & & & & \\
\hline 0.2 & 0.99232 & $(0.99233)$ & 0.99310 & (0.99311) & 1.00076 & (1.00076) & 0.99437 & (0.99437) \\
\hline 0.4 & 0.94181 & $(0.94257)$ & 0.94775 & $(0.94842)$ & 1.00582 & $(1.00514)$ & 0.95737 & $(0.95784)$ \\
\hline 0.6 & 0.81559 & $(0.82975)$ & 0.83379 & $(0.84624)$ & 1.01279 & (0.99996) & 0.86334 & $(0.87227)$ \\
\hline 0.8 & 0.57210 & $(0.67553)$ & 0.60930 & $(0.70219)$ & 0.98474 & $(0.91849)$ & 0.67050 & $(0.74153)$ \\
\hline 0.9 & 0.36698 & $(0.60439)$ & 0.41151 & $(0.63192)$ & 0.85040 & $(0.81012)$ & 0.48386 & $(0.66867)$ \\
\hline 0.95 & 0.21849 & $(0.57587)$ & 0.25896 & $(0.60203)$ & 0.62403 & $(0.73151)$ & 0.32181 & $(0.63354)$ \\
\hline 0.99 & 0.05313 & $(0.55808)$ & 0.07050 & $(0.58216)$ & 0.18838 & $(0.65559)$ & 0.09416 & $(0.60726)$ \\
\hline 0.995 & 0.02742 & & 0.03765 & & 0.10045 & & 0.05103 & \\
\hline \multirow[t]{2}{*}{0.999} & 0.00566 & & 0.00813 & & 0.02130 & & 0.01120 & \\
\hline & $\zeta e / k T=$ & & & & & & & \\
\hline 0.2 & 0.99436 & (0.99436) & 1.01004 & (1.01004) & 0.99600 & $(0.99600)$ & 0.87160 & (0.87164) \\
\hline 0.4 & 0.95735 & $(0.95790)$ & 1.07979 & (1.07948) & 0.96988 & (0.97024) & 0.00308 & (0.01193) \\
\hline 0.6 & 0.86336 & $(0.87387)$ & 1.26036 & $(1.25681)$ & 0.90193 & $(0.90874)$ & -2.20226 & $(-2.05070)$ \\
\hline 0.8 & 0.66984 & $(0.75174)$ & 1.56955 & (1.58573) & 0.74932 & $(0.80875)$ & -6.16959 & $(-5.35636)$ \\
\hline 0.9 & 0.48527 & $(0.68985)$ & 1.70590 & (1.85084) & 0.58045 & $(0.74960)$ & -8.61952 & $(-7.54210)$ \\
\hline 0.95 & 0.32920 & $(0.66306)$ & 1.64942 & (2.03081) & 0.41533 & $(0.72061)$ & -9.28579 & $(-8.85757)$ \\
\hline 0.99 & 0.10698 & $(0.64524)$ & 1.16253 & (2.20893) & 0.14498 & $(0.69912)$ & -7.15545 & $(-10.0748)$ \\
\hline 0.995 & 0.06133 & & 0.93865 & & 0.08451 & & -5.85974 & \\
\hline 0.999 & 0.01523 & & 0.53734 & & 0.02147 & & -3.4055 & \\
\hline
\end{tabular}

Note. The figures in parentheses are asymptotic solutions calculated using Eq. (A.38).

Some numerical solutions for the normalized diffusiophoretic velocity $U / U_{0}$ of a spherical particle normal to a plane wall as functions of $a / b$ are depicted in Fig. 2 for various values of the parameters $\alpha$ and $\kappa a$. It can be seen that $U / U_{0}$ decreases with an increase in $\kappa a$ for the otherwise specified condition. This behavior is expected knowing that the electrochemical potential gradients on the particle surface near a normal plane wall decrease as $\kappa a$ (or $G$ ) increases (see the analysis in Appendix A). Under the situation of large $\kappa a$, the diffusiophoretic mobility of the particle near the plane wall is 


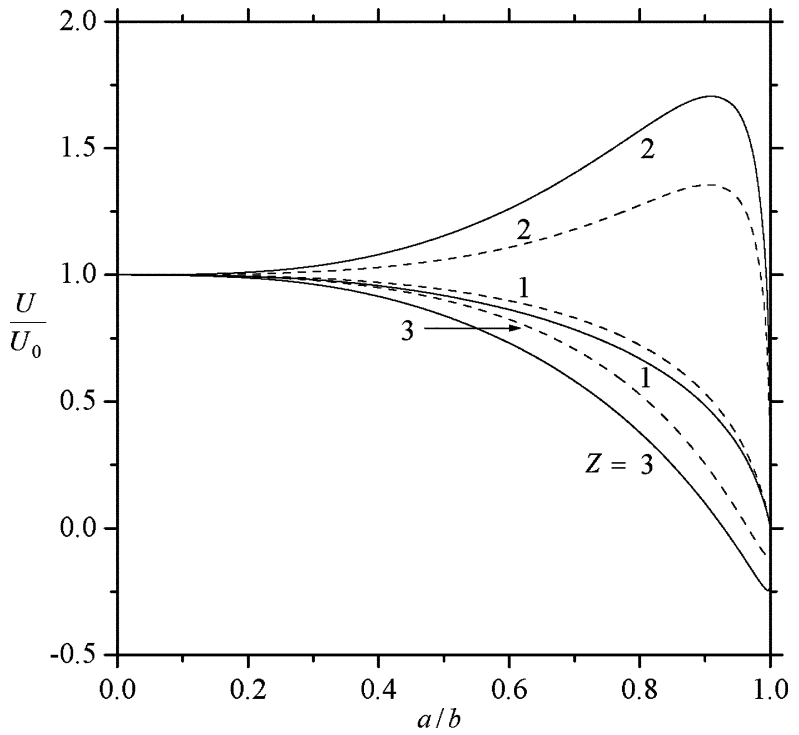

(a)

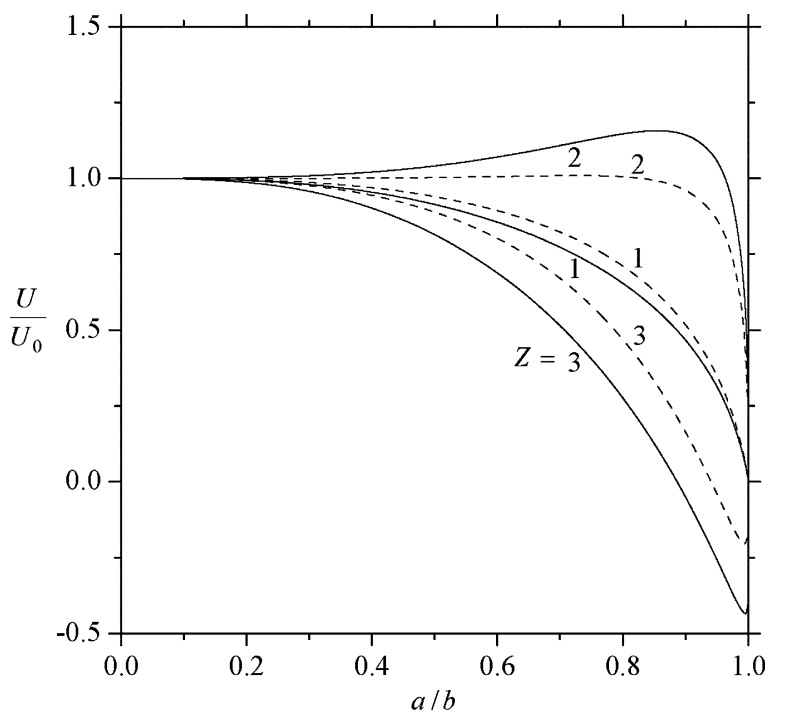

(b)

Fig. 3. Plots of the normalized diffusiophoretic velocity $U / U_{0}$ of a spherical particle perpendicular to two plane walls with $b /(b+c)=0.5$ (solid curves) and $b /(b+c)=0.25$ (dashed curves) versus the separation parameter $a / b$ with $f_{1}=0.2$ and $\kappa a=100$ for various values of $Z:$ (a) $\alpha=0$ and $\zeta e / k T= \pm 5$; (b) $\alpha=-0.2$ and $\zeta e / k T=-5$.

a monotonic decreasing function of $a / b$. In the limiting case of $\kappa a \rightarrow \infty$, the diffusiophoretic mobility of the particle is identical to the prediction by ignoring the polarization effect of the diffuse layer [20]. On the contrary, under the situation of relatively small $\kappa a$ (e.g., with $\kappa a=20$ ), the diffusiophoretic mobility of the particle increases with an increase in $a / b$ as $a / b$ is small or moderate, but decreases from a maximum with increasing $a / b$ as $a / b$ is sufficiently large. This interesting feature that $U / U_{0}$ may not be a monotonic decreasing function of $a / b$ and can even be greater than unity is understood because the wall effect of hydrodynamic resistance on the particle is in competition with the wall effect of electrochemical enhancement when a particle with small $\kappa a$ is undergoing diffusiophoretic motion normal to a plane wall.

\subsection{Motion perpendicular to two plane walls}

The diffusiophoretic velocity of a dielectric sphere normal to two plane wall is a complicated function of the properties of the particle and suspending solution as well as the relative separation distances. In Table 2, the collocation solutions for the normalized velocity $U / U_{0}$ of a spherical particle located between two parallel plane walls whose distance to one wall is the same as to the other (with $c=b$ ) undergoing diffusiophoresis perpendicularly are presented for various values of the parameters $\alpha, Z, \zeta e / k T$, and $a / b$. The corresponding methodof-reflection solutions, given by Eq. (A.38) in Appendix A as a power series expansion in $\lambda(=a / b)$ correct to $O\left(\lambda^{8}\right)$, are also listed in this table for comparison. Similar to the case of diffusiophoresis of a spherical particle normal to a single plane wall considered in the previous subsection, the approximate analytical formula (A.38) agrees quite well with the collocation results as long as $\lambda<0.6$, but can have significant errors when $\lambda>0.6$. A comparison between Table 2 for the case of a slit and Table 1 for the case of a single normal plane indicates that the assumption that the boundary effect for two walls can be obtained by simple addition of single-wall effects can lead to a greater or smaller correction to diffusiophoretic motion, depending on the combination of the relevant parameters.

Numerical results of the normalized diffusiophoretic velocity $U / U_{0}$ of a spherical particle perpendicular to two plane walls at two particular positions between them (with $b /(b+c)=0.25$ and 0.5$)$ obtained using the boundarycollocation method are plotted as functions of $a / b$ and $Z$ in Fig. 3 with $f_{1}=0.2$ and $\kappa a=100$. For the case of $\alpha=0$ $\left(\bar{D}_{1}=\bar{D}_{2}\right)$, the macroscopic electric field induced by the electrolyte concentration gradient vanishes and the particle velocity, which is due to the chemiphoretic effect only, is an even function of zeta potential. It can be seen that, for a specified value of $b /(b+c), U / U_{0}$ is not necessarily a monotonic function of $a / b$. For some cases, the particle will reverse the direction of diffusiophoresis and the magnitude of its normalized velocity can be dramatically varied when the separation distance between the particle and the plane walls is changed. In general, no simple rule could appropriately predict the boundary effect on diffusiophoresis. As expected, for a given value of $a / b$, the boundary effect on the diffusiophoretic particle is stronger for the case of $b /(b+c)=0.5$ than that of $b /(b+c)=0.25$. Although it is difficult to obtain convergent results of the particle velocity for situations of very small separation distances $(a / b>0.999)$, both physical reasoning and numerical tendency indicate that $U / U_{0} \rightarrow 0$ as $a / b \rightarrow 1$.

In Figs. 4 and 5, the collocation solutions for the normalized velocity $U / U_{0}$ of a spherical particle situated midway between two parallel plane walls (with $c=b$ ) undergoing diffusiophoresis perpendicularly are plotted versus $\zeta e / k T$ in the range from -8 to 8 for cases of $\alpha=0$ and -0.2 , respectively, with $a / b=0.6$ and various values of $\kappa a$. Note that $U=U_{0}=0$ 


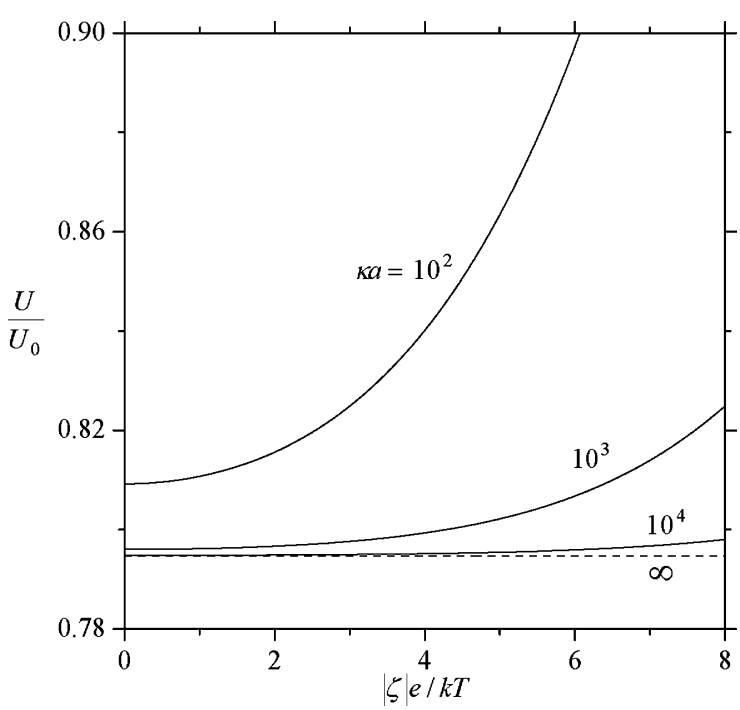

(a)

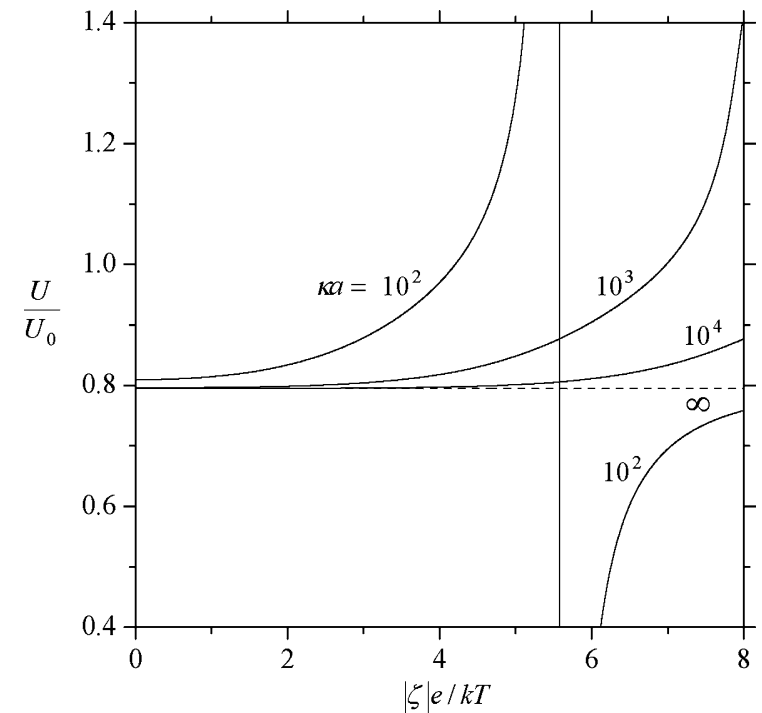

(b)

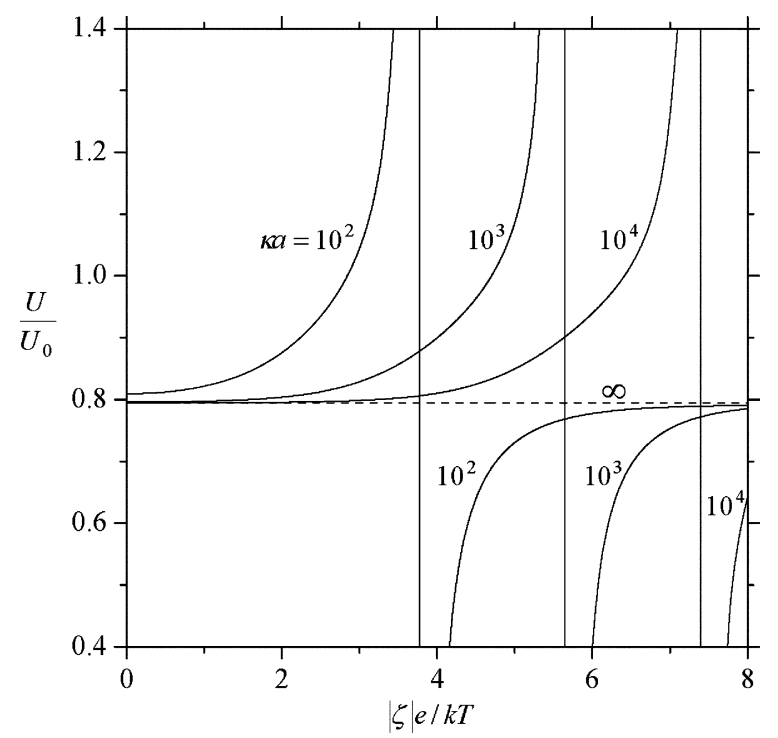

(c)

Fig. 4. Plots of the normalized diffusiophoretic velocity $U / U_{0}$ of a spherical particle perpendicular to two equally distant plane walls $(c=b)$ versus $\zeta e / k T$ with $a / b=0.6$ and $f_{1}=f_{2}=0.2(\alpha=0)$ for various values of $\kappa a$ : (a) $Z=1$; (b) $Z=2$; (c) $Z=3$.

at $\zeta e / k T=0$. As expected, when the value of $\kappa a$ is large and the magnitude of $\zeta e / k T$ is small to satisfy Eq. (6), the diffusiophoretic mobility of the particle approaches the value calculated by ignoring the polarization effect of the double layers (shown by a dashed line labeled with $\kappa a \rightarrow \infty$ in each figure). These figures illustrate that the difference of the results obtained from the presence and absence of the polarization effect can be quite substantial and needs to be considered. For given values of $Z$ and $\kappa a$, there can be a maximum and a minimum of the normalized particle mobility occurring at an associated value of $\zeta e / k T$ (in addition to the one with small magnitude appearing for the case of $\alpha \neq 0$ ). If the value of $\kappa a$ is smaller or the counterions have a larger magnitude of valence, the locations of these extremes will shift toward a smaller magnitude of $\zeta e / k T$. Because the direction of diffusiophoresis of an isolated spherical particle can reverse with the variation of $Z, \zeta e / k T$, or $\kappa a$
[11-13], these extremes arise at combinations of $Z, \zeta e / k T$, and $\kappa a$ in which $U_{0} \rightarrow 0$.

A careful comparison of the results in Figs. 3-5 (or Table 2) for the case of a slit with those in Fig. 2 (or Table 1) for the case of a single wall reveals an interesting feature of the boundary effect on diffusiophoresis of a colloidal sphere. The presence of a second, identical, normal plane wall, even at a symmetric position with respect to the sphere against the first, does not always enhance the wall effect on the diffusiophoretic particle induced by the first plane only. This outcome reflects again the fact that the confining wall can affect the electrochemical driving force and the viscous drag force on a particle in opposite directions. Each force is increased in its own direction as the value of $a / b$ turns large, but to a different degree, for the case of diffusiophoretic motion of a particle in a slit relative to that for the case of migration normal to a single plate. Thus, the net effect 


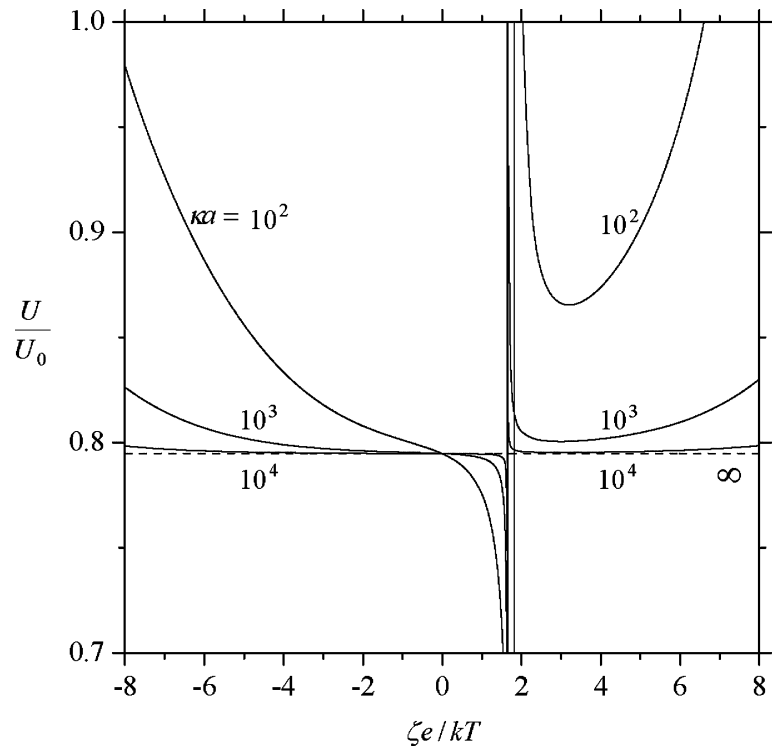

(a)

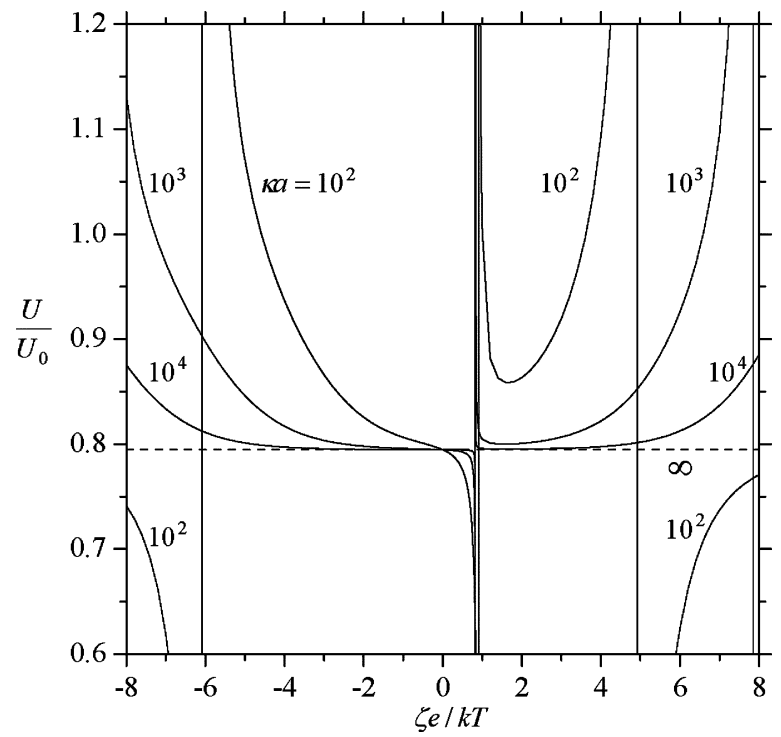

(b)

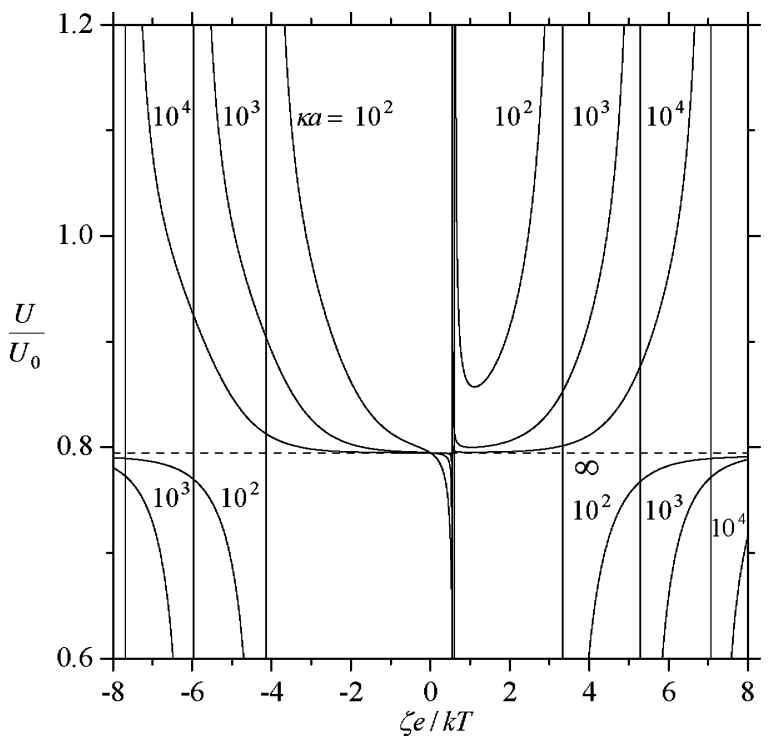

(c)

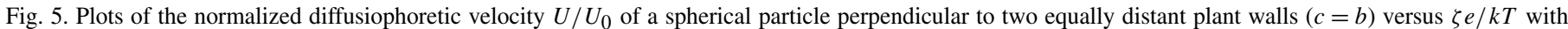
$a / b=0.6, f_{1}=0.2$ and $\alpha=-0.2$ for various values of $\kappa a$ : (a) $Z=1$; (b) $Z=2$; (c) $Z=3$.

composed of these two opposite forces for the slit case is not necessarily to enhance that for the case of a single wall.

In Fig. 6, the collocation results for the normalized diffusiophoretic velocity $U / U_{0}$ of a charged sphere normal to two plane walls at various positions between them are plotted for some cases. The dashed curves (with $a / b=$ constant) illustrate the effect of the position of the second wall (at $z=c$ ) on the particle velocity for various values of the relative sphere-to-first-wall spacing $b / a$. The solid curves [with $2 a /(b+c)=$ constant] indicate the variation of the particle velocity as a function of the sphere position at various values of the relative wall-to-wall spacing $(b+c) / 2 a$. It can be seen that the net wall effect can reduce or enhance the diffusiophoretic mobility $U / U_{0}$ of the particle. At a constant value of $2 a /(b+c)$, the particle experiences a minimum viscous drag force and in general has a greatest velocity when it is located midway between the two walls (with $c=b$ ), as indicated in the cases of Figs. 6a and 6c; the hydrodynamic drag increases and the diffusiophoretic velocity decreases as the particle approaches either of the walls [or the ratio $b /(b+c)$ decreases]. Interestingly, as illustrated in Fig. 6a, at some specified values of $a / b$ for the diffusiophoretic particle near a first wall, the presence and approach of a second plate can increase the velocities of the particle when it is far from the particle ( $c$ is large), and then reduce the particle velocity when it is close to the particle. On the other hand, for the case shown in Fig. 6b, the net wall effect is to increase the diffusiophoretic mobility $U / U_{0}$ of the particle; at a constant value of $2 a /(b+c)$, the normalized particle mobility has a smallest value when it is located midway between the two walls, where the particle experiences a minimum effect of electrochemical 


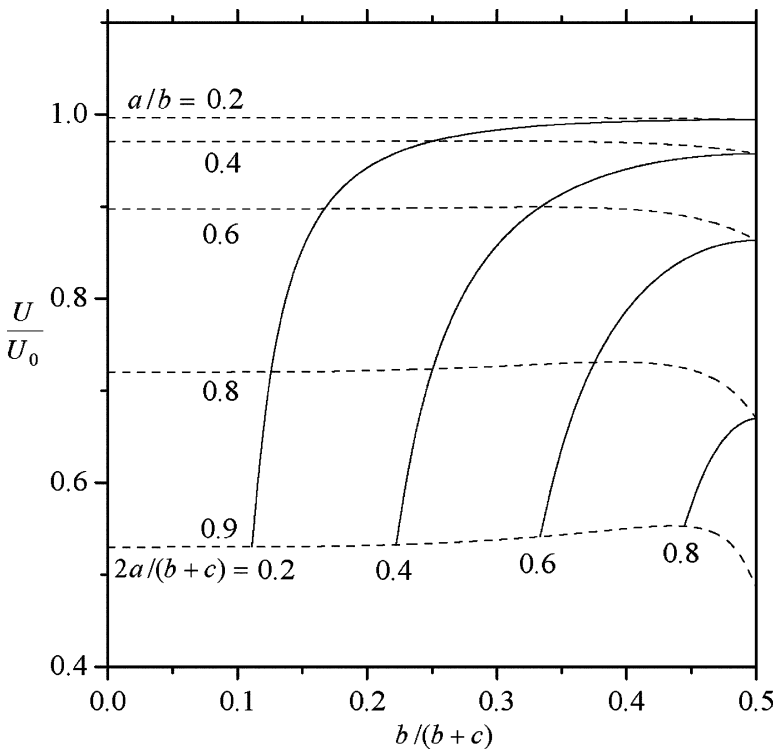

(a)

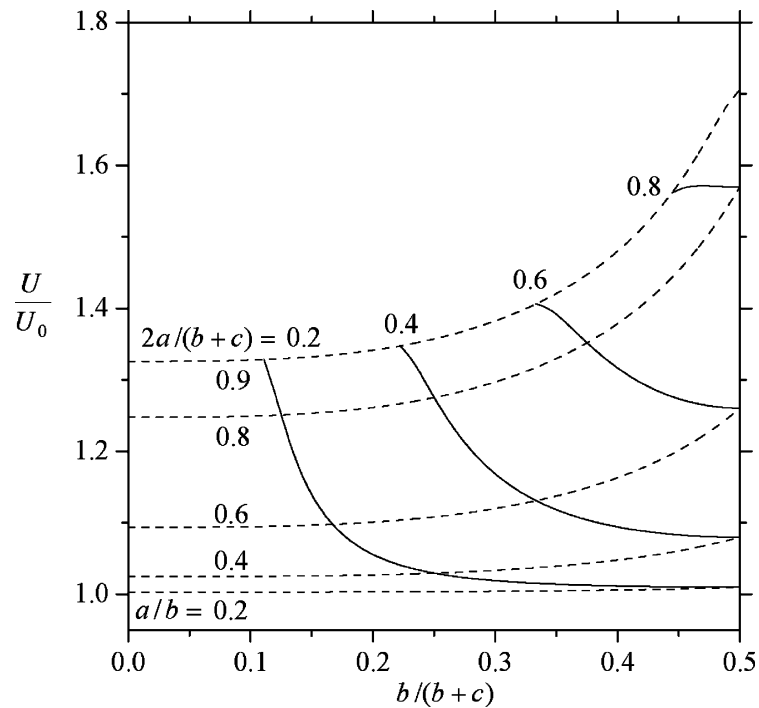

(b)

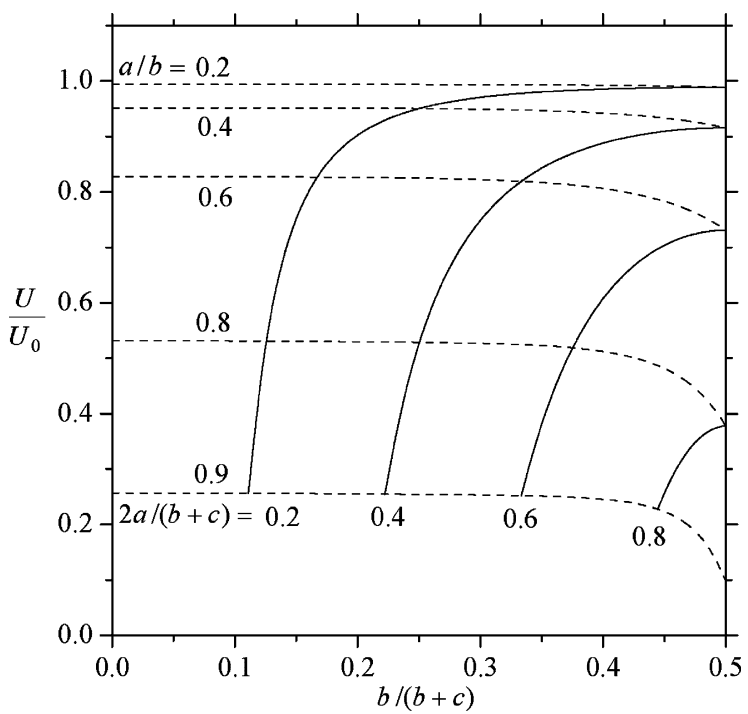

(c)

Fig. 6. Plots of the normalized diffusiophoretic velocity $U / U_{0}$ of a spherical particle perpendicular to two plane walls versus $b /(b+c)$ with $a / b$ and $2 a /(b+c)$ as parameters at $f_{1}=f_{2}=0.2(\alpha=0), \zeta e / k T= \pm 5$, and $\kappa a=100$ : (a) $Z=1$; (b) $Z=2$; (c) $Z=3$.

enhancement, and becomes larger when it approaches either of the walls.

\section{Electrophoresis}

Considered in this section is the quasisteady electrophoretic motion of a dielectric sphere in a uniformly applied electric field $\mathbf{E}^{\infty}=E^{\infty} \mathbf{e}_{z}$ perpendicular to two large plane walls at an arbitrary position between them. The velocity of the particle caused by the field is $\mathbf{U}=U \mathbf{e}_{z}$. The bulk concentration $n^{\infty}$ of the symmetric electrolyte in the fluid beyond the electric double layer is constant now. Like the analysis in Section 2, the thickness of the double layer is assumed to be much smaller than the radius of the particle and the surface-to-surface distance between the particle and each wall, but the polarization effect in the thin diffuse layer is incorporated.
Outside the double layer, the electrochemical potentials $\mu_{m}$ of the ions still satisfy Laplace's equation (8) and boundary conditions (10)-(13), but their undisturbed values in Eq. (14) are replaced by

$\mu_{m \infty}=\mu_{m}^{0}+k T \ln n^{\infty}-(-1)^{m} Z e E^{\infty} z$.

The solution for $\mu_{m}$ in this case can still be expressed as Eq. (18) with coefficients $R_{m n}$ determined by

$$
\begin{aligned}
& \sum_{n=0}^{\infty} \sum_{m=1}^{2} R_{m n} R_{m}^{\prime}(\theta)=Z e E^{\infty}\left(1-\frac{2 \beta_{11}}{a}+\frac{2 \beta_{12}}{a}\right) \cos \theta, \\
& \sum_{n=0}^{\infty} \sum_{m=1}^{2} R_{m n} R_{m}^{\prime \prime}(\theta)=-Z e E^{\infty}\left(1+\frac{2 \beta_{21}}{a}-\frac{2 \beta_{22}}{a}\right) \cos \theta,
\end{aligned}
$$


Table 3

Normalized electrophoretic velocity of a dielectric sphere perpendicular to a single plane wall (with $c \rightarrow \infty$ ) obtained from the boundary-collocation method for the case $f_{1}=f_{2}=0.2$ and $\kappa a=100$

\begin{tabular}{|c|c|c|c|c|}
\hline \multirow[t]{2}{*}{$a / b$} & \multicolumn{4}{|l|}{$U / U_{0}$} \\
\hline & $Z=1$ & & $Z=2$ & \\
\hline & $\zeta e / k T=2$ & & & \\
\hline 0.2 & 0.99511 & $(0.99511)$ & 0.99528 & $(0.99528)$ \\
\hline 0.4 & 0.96076 & $(0.96073)$ & 0.96224 & $(0.96220)$ \\
\hline 0.6 & 0.86075 & $(0.86192)$ & 0.86633 & $(0.86686)$ \\
\hline 0.8 & 0.62218 & $(0.64915)$ & 0.63725 & $(0.65814)$ \\
\hline 0.9 & 0.39379 & $(0.48486)$ & 0.41460 & $(0.49218)$ \\
\hline 0.95 & 0.22716 & $(0.38791)$ & 0.24713 & $(0.39164)$ \\
\hline 0.99 & 0.05222 & $(0.30429)$ & 0.06084 & $(0.30294)$ \\
\hline 0.995 & 0.02666 & & 0.03169 & \\
\hline \multirow[t]{2}{*}{0.999} & 0.00540 & & 0.00660 & \\
\hline & $\zeta e / k T=5$ & & & \\
\hline 0.2 & 0.99557 & $(0.99557)$ & 0.99639 & (0.99639) \\
\hline 0.4 & 0.96460 & $(0.96453)$ & 0.97135 & $(0.97124)$ \\
\hline 0.6 & 0.87502 & $(0.87475)$ & 0.89916 & $(0.89788)$ \\
\hline 0.8 & 0.66040 & $(0.67333)$ & 0.72497 & $(0.72654)$ \\
\hline 0.9 & 0.44731 & $(0.50674)$ & 0.54830 & $(0.57710)$ \\
\hline 0.95 & 0.28047 & $(0.40243)$ & 0.40387 & $(0.47989)$ \\
\hline 0.99 & 0.07873 & $(0.30794)$ & 0.19900 & $(0.38934)$ \\
\hline 0.995 & 0.04327 & & 0.14864 & \\
\hline 0.999 & 0.0100 & & 0.0774 & \\
\hline
\end{tabular}

Note. The figures in parentheses are asymptotic solutions calculated using Eq. (A.28).

to replace Eq. (19). The governing equation, boundary conditions, and solution for the fluid velocity field have the same forms as those given by Eqs. (21)-(31). The final results for the electrophoretic velocity of the particle can be determined by the simultaneous solution of Eqs. (32) and (35), with

$F_{m}(\theta)=(-1)^{m} Z e E^{\infty} \sin \theta+\sum_{n=0}^{\infty} R_{m n} \delta_{n}^{(3)}(a, \theta)$,

in replacement of Eq. (33).

Some converged collocation solutions for the electrophoretic velocity of a dielectric sphere (which is an odd function of the parameter $\zeta e / k T$ ) normal to a plane wall (with $c \rightarrow \infty$ ) for various values of $a / b$ are presented in Table 3 , while the corresponding results for the electrophoretic velocity of the particle located at the median plane between two parallel plates (with $c=b$ ) are given in Table 4. Now, the electrophoretic velocity of the particle in an unbounded fluid given by Eq. (5) is used to normalize the wall-corrected values. The corresponding method-of-reflection solutions, given by Eqs. (A.28) and (A.38) with the parameters defined by Eqs. (A.29) and (A.30) in Appendix $\mathrm{A}$ as power series expansions in $\lambda(=a / b)$ correct to $O\left(\lambda^{8}\right)$, are also listed in these tables for comparison. Analogous to the cases of diffusiophoresis considered in the previous section, the asymptotic formulas (A.28) and (A.38) for $U / U_{0}$ agree quite well with the collocation results as long as $\lambda \leqslant 0.6$, but can have significant errors for greater values of $\lambda$.

The collocation results for the normalized electrophoretic mobility $U / U_{0}$ of a spherical particle normal to a plane wall and the corresponding results for the particle undergoing electrophoresis at the median plane between two parallel plane
Table 4

Normalized electrophoretic velocity of a dielectric sphere perpendicular to two equally distant plane walls (with $c=b$ ) obtained from the boundary-collocation method for the case $f_{1}=f_{2}=0.2$ and $\kappa a=100$

\begin{tabular}{|c|c|c|c|c|}
\hline \multirow[t]{2}{*}{$a / b$} & \multicolumn{4}{|l|}{$U / U_{0}$} \\
\hline & $Z=1$ & & $Z=2$ & \\
\hline & $\zeta e / k T=2$ & & & \\
\hline 0.2 & 0.99158 & (0.99159) & 0.99200 & (0.99201) \\
\hline 0.4 & 0.93621 & $(0.93710)$ & 0.93940 & $(0.94023)$ \\
\hline 0.6 & 0.79834 & (0.81487) & 0.80813 & $(0.82362)$ \\
\hline 0.8 & 0.53601 & $(0.65430)$ & 0.55612 & $(0.66803)$ \\
\hline 0.9 & 0.32471 & $(0.58644)$ & 0.34864 & $(0.60002)$ \\
\hline 0.95 & 0.18305 & $(0.56232)$ & 0.20437 & $(0.57471)$ \\
\hline 0.99 & 0.04132 & $(0.54962)$ & 0.04997 & $(0.56043)$ \\
\hline 0.995 & 0.02105 & & 0.02605 & \\
\hline 0.999 & 0.00429 & & 0.00547 & \\
\hline & $\zeta e / k T=5$ & & & \\
\hline 0.2 & 0.99268 & (0.99268) & 0.99465 & $(0.99465)$ \\
\hline 0.4 & 0.94456 & $(0.94532)$ & 0.96010 & $(0.96081)$ \\
\hline 0.6 & 0.82400 & $(0.83831)$ & 0.87544 & $(0.88916)$ \\
\hline 0.8 & 0.58870 & $(0.69370)$ & 0.70927 & $(0.81423)$ \\
\hline 0.9 & 0.38813 & $(0.62920)$ & 0.55821 & $(0.80712)$ \\
\hline 0.95 & 0.24141 & $(0.60478)$ & 0.43411 & $(0.82104)$ \\
\hline 0.99 & 0.06839 & $(0.59083)$ & 0.23830 & $(0.84422)$ \\
\hline 0.995 & 0.03783 & & 0.18331 & \\
\hline 0.999 & 0.00892 & & 0.09930 & \\
\hline
\end{tabular}

Note. The figures in parentheses are asymptotic solutions calculated using Eq. (A.38)

walls in the normal direction as functions of $a / b$ are plotted in Fig. 7 for various values of $\kappa a$ and $\zeta e / k T\left(U / U_{0}\right.$ is an even function of $\zeta e / k T)$. In either case, the value of $U / U_{0}$ is a monotonic decreasing function of $a / b$. It appears that $U / U_{0}$ decreases with an increase in $\kappa a$ and with a decrease in the magnitude of $\zeta e / k T$. As expected, the presence of a second normal plane wall in general enhances the wall effect on the electrophoretic particle induced by the first plate only.

In Fig. 8, the normalized electrophoretic mobility $U / U_{0}$ of a spherical particle at the median plane between two parallel plane walls in the normal direction is plotted versus the particle's zeta potential for three cases of $\kappa a$ when the separation parameter $a / b$ is kept constant. When $Z=1$, as shown in Fig. 8a, $U / U_{0}$ is a monotonic increasing function of the magnitude of the nondimensional zeta potential $\zeta e / k T$ ranging from 0 to 8 and the boundary effect is weakened $\left(U / U_{0}\right.$ is larger) as the value of $\kappa a$ becomes smaller. However, when $Z=2$ or 3 as illustrated in Figs. 8b and 8c, a maximum of $U / U_{0}$ may exist at a magnitude of $\zeta e / k T$ for a specified $\kappa a$ or at a value of $\kappa a$ for a given $\zeta e / k T$. If the specified $\kappa a$ increases, the maximum occurs at a larger magnitude of $\zeta e / k T$; if the magnitude of the given $\zeta e / k T$ increases, the maximum occurs at a larger $\kappa a$. If the counterions have a larger magnitude of valence, the location of this maximum will shift toward a smaller magnitude of $\zeta e / k T$ or larger $\kappa a$. Similar to the case of diffusiophoresis considered in the previous section, no simple rule could appropriately describe the boundary effects on the electrophoretic mobility of the particle, which are dependent on the combination of $\zeta e / k T, \kappa a, Z, f_{1}, f_{2}$, and $a / b$.

In Fig. 9, collocation results for the normalized electrophoretic velocity $U / U_{0}$ of a dielectric sphere perpendicular 


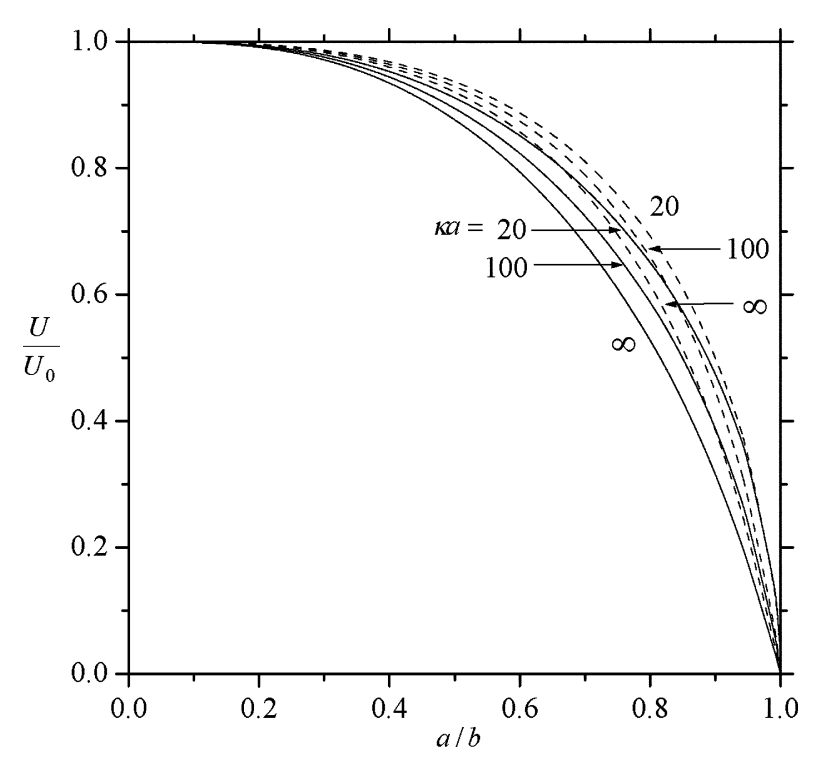

(a)

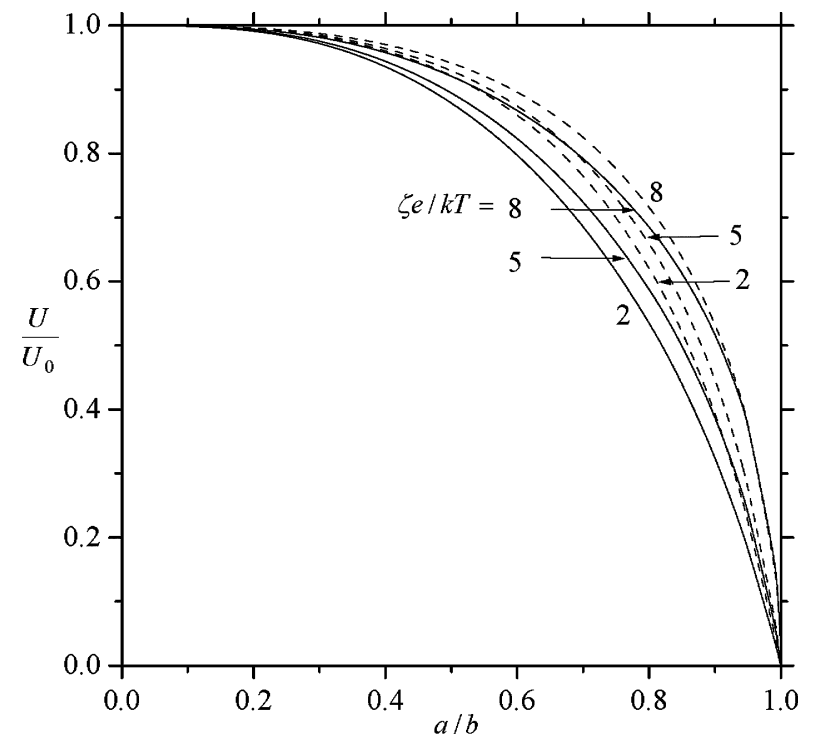

(b)

Fig. 7. Plots of the normalized electrophoretic velocity $U / U_{0}$ of a spherical particle perpendicular to two plane walls (solid curves) and to a single plane wall (dashed curves) versus the separation parameter $a / b$ with $Z=1$ and $f_{1}=f_{2}=0.2$ : (a) $\zeta e / k T= \pm 5$; (b) $\kappa a=100$.

to two plane walls at various positions between them are displayed. It can be seen that the particle experiences a minimum viscous drag and has a greatest electrophoretic velocity when it is located midway between the two walls (with $c=b$ ). At a relatively small value of $a / b$ for the electrophoretic particle near a first normal wall, the presence of a second plate is to further reduce the velocity of the particle, and the degree of this reduction increases monotonically with a decrease in the relative distance between the particle and the second plate [or with an increase in $b /(b+c)]$. For a specified value of $a / b$ greater than about 0.8 , however, the presence of a second plane wall is not necessarily to further reduce the particle velocity.

\section{Conclusions}

The numerical solutions and approximate analytical solutions for the quasisteady diffusiophoretic and electrophoretic motions of a charged sphere perpendicular to two infinite plane walls at an arbitrary position between them have been obtained in this work by using the boundary-collocation technique and the method of reflections, respectively, in the limit of vanishingly small Reynolds and Peclet numbers. It has been found that the boundary effects on these phoretic motions of a particle are quite significant and are complicated functions of the properties of the particle and surrounding ions $\left(\zeta e / k T, \kappa a, Z, f_{1}\right.$, and $\left.f_{2}\right)$ and the separation distances $(a / b$ and $a / c)$. The diffusiophoretic or electrophoretic mobility of a particle near a wall is generally, but not necessarily, a monotonic decreasing function of the separation parameter $a / b$. When the value of $a / b$ is sufficiently large, the effect of a confining wall can speed up or slow down the particle velocity relative to its isolated value depending on the values of the relevant parameters of the particle-electrolyte system. This behavior reflects the competition between the relatively weak hydrodynamic retardation exerted by the neighboring wall on the particle migration and the possible, strong phoretic enhancement due to the electrochemical interaction between the particle and the wall. No general rule can make an adequate prediction for such complicated phenomena present in the boundary effects on diffusiophoresis and electrophoresis.

The diffusiophoretic and electrophoretic mobilities of a spherical particle parallel to two infinite plane walls at an arbitrary position between them were calculated in a previous work [26] for various values of the parameters $\alpha, \zeta e / k T, \kappa a$, $a / b$, and $b /(b+c)$. It was also found that the particle mobilities may decrease or increase with increasing $a / b$ and the particle can migrate faster than it would as $a / b=0$, depending the electrochemical boundary condition at the walls and the combination of the relevant parameters. The effect of viscous retardation is stronger and the effect of possible electrochemical enhancement is weaker in a transverse diffusiophoresis or electrophoresis than in a parallel motion. In general, the net boundary effects on diffusiophoresis and electrophoresis of a particle are stronger for the perpendicular migration. For the general problem of a particle undergoing diffusiophoresis or electrophoresis in an arbitrary direction with respect to the two parallel plane walls, the solution can be obtained by adding both the parallel and the transverse results vectorially.

\section{Acknowledgment}

Part of this research was supported by the National Science Council of the Republic of China.

\section{Appendix A. Analysis of the diffusiophoresis and electrophoresis of a spherical particle perpendicular to one or two plane walls by a method of reflections}

In this appendix, the quasisteady diffusiophoretic and electrophoretic motions of a dielectric sphere in the solution of a 


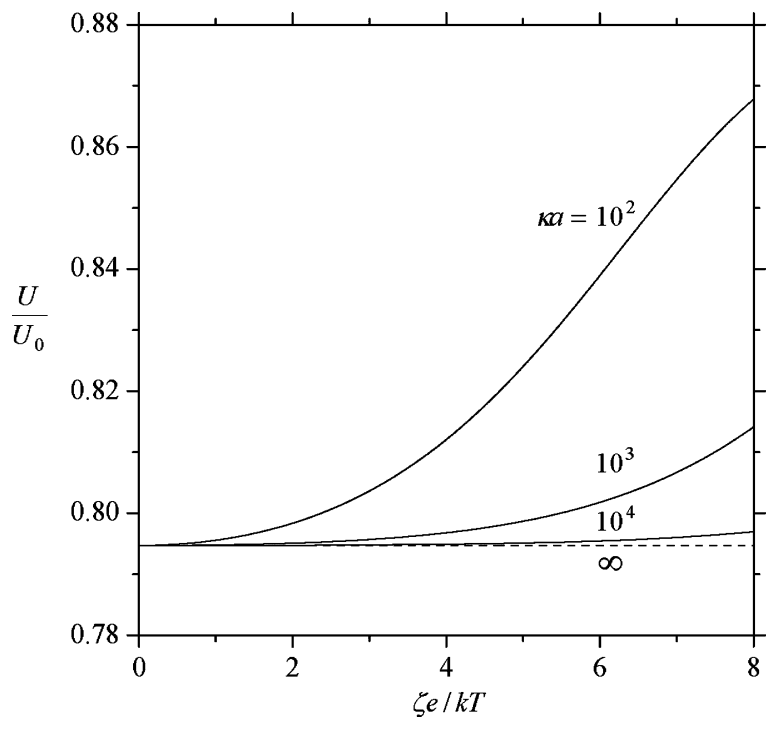

(a)

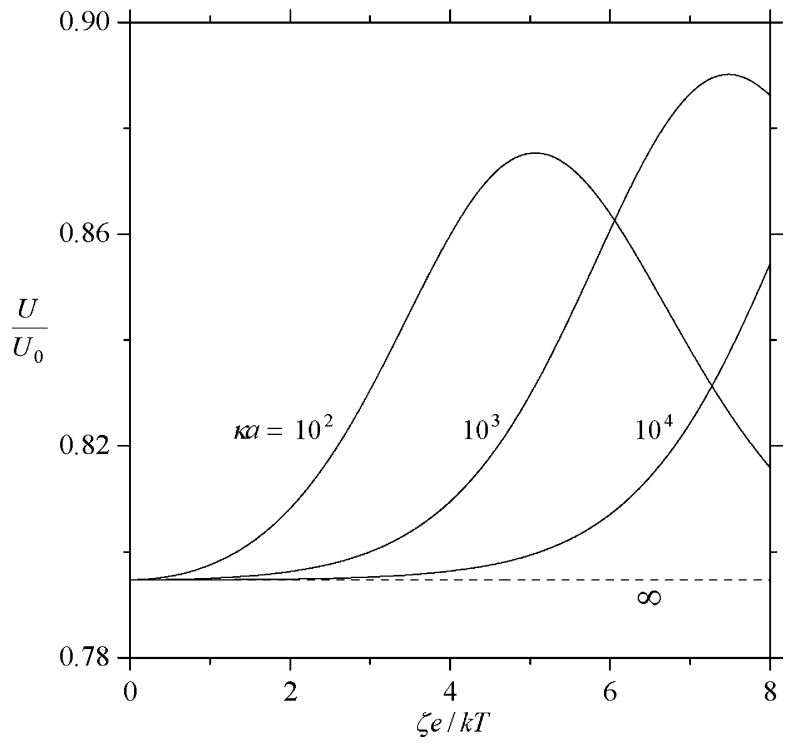

(b)

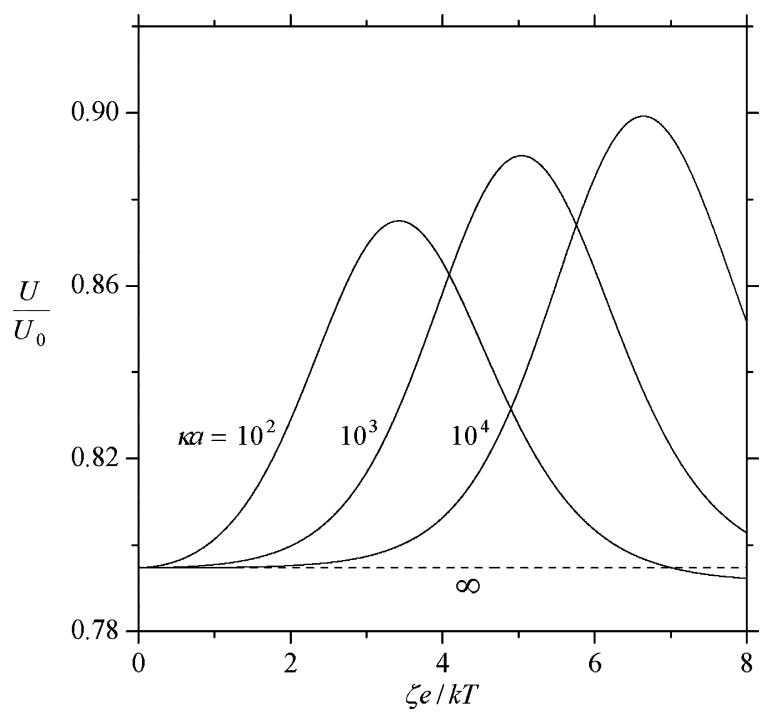

(c)

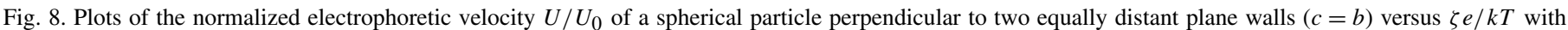
$a / b=0.6$ and $f_{1}=f_{2}=0.2$ for various values of $\kappa a$ : (a) $Z=1$; (b) $Z=2$; (c) $Z=3$.

symmetric electrolyte perpendicular either to an infinite plane wall (with $c \rightarrow \infty$ ) or to two parallel plane walls with equal distances from the particle $(c=b)$, as shown in Fig. 1, will be analyzed using a method of reflections. The effect of the walls on the particle velocity $\mathbf{U}$ is sought in expansions of $\lambda$, which equals $a / b$, the ratio of the particle radius to the distance between the particle center and the walls.

\section{A.1. Motion normal to a single plane wall}

For the problem of diffusiophoretic motion of a spherical particle driven by a uniform electrolyte concentration gradient $\nabla n^{\infty}$ normal to an infinite plane wall, the governing equations (8) and (21) must be solved by satisfying the boundary conditions (10), (12), (13), and (24)-(26) with $c \rightarrow \infty$. The method-of-reflection solution for the ionic electrochemical po- tential and velocity fields in the fluid phase consists of the following series, whose terms depend on increasing powers of $\lambda$,

$\mu_{m}=\mu_{m \infty}+\mu_{m p}^{(1)}+\mu_{m \mathrm{w}}^{(1)}+\mu_{m \mathrm{p}}^{(2)}+\mu_{m \mathrm{w}}^{(2)}+\cdots$,

$\mathbf{v}=\mathbf{v}_{\mathrm{p}}^{(1)}+\mathbf{v}_{\mathrm{w}}^{(1)}+\mathbf{v}_{\mathrm{p}}^{(2)}+\mathbf{v}_{\mathrm{w}}^{(2)}+\cdots$,

where the subscripts $\mathrm{p}$ and $\mathrm{w}$ represent the reflections from the particle and wall, respectively, and the superscript (i) denotes the $i$ th reflection from that surface. In these series, all the expansion sets of the electrochemical potential and velocity fields must satisfy Eqs. (8) and (21).

According to Eq. (A.1), the diffusiophoretic velocity of the particle can also be expressed in the series form,

$\mathbf{U}=U_{0} \mathbf{e}_{z}+\mathbf{U}^{(1)}+\mathbf{U}^{(2)}+\cdots$. 


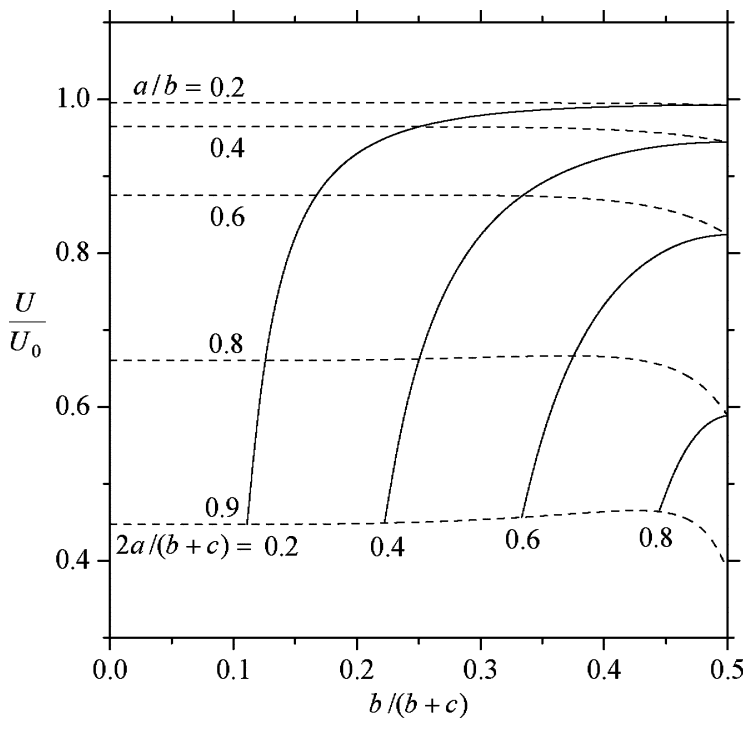

(a)

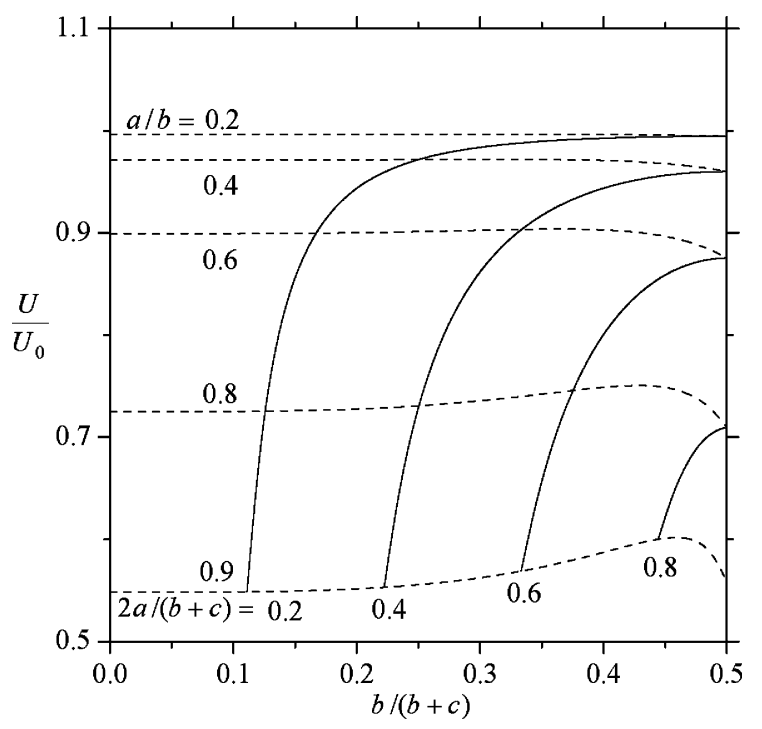

(b)

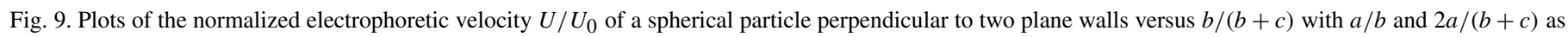
parameters at $f_{1}=f_{2}=0.2, \zeta e / k T= \pm 5$, and $\kappa a=100$ : (a) $Z=1$; (b) $Z=2$.

In this expression, $U_{0}$ is the diffusiophoretic velocity of an identical particle in the corresponding unbounded continuous phase given by Eq. (7); $\mathbf{U}^{(i)}$ is related to $\nabla \mu_{m \mathrm{w}}^{(i)}$ and $v_{\mathrm{w}}^{(i)}$ by the Faxen law [28,29],

$\mathbf{U}^{(i)}=\sum_{m=1}^{2} G_{m}\left[\nabla \mu_{m \mathrm{w}}^{(i)}\right]_{0}+\left[\mathbf{v}_{\mathrm{w}}^{(i)}\right]_{0}+\frac{a^{2}}{6}\left[\nabla^{2} \mathbf{v}_{\mathrm{w}}^{(i)}\right]_{0}$,

where the subscript 0 to variables inside brackets denotes evaluation at the position of the particle center. In Eq. (A.3),

$G_{1}=\frac{4 \varepsilon k T}{3 \eta(Z e)^{2}}\left[\left(1+g_{11}-g_{21}\right) \bar{\zeta}+\left(1+g_{11}+g_{21}\right) \ln \cosh \bar{\zeta}\right]$,

$G_{2}=\frac{4 \varepsilon k T}{3 \eta(Z e)^{2}}\left[\left(-1+g_{12}-g_{22}\right) \bar{\zeta}+\left(1+g_{12}+g_{22}\right) \ln \cosh \bar{\zeta}\right]$

where $\bar{\zeta}$ is defined by Eq. (4), and

$g_{11}=\frac{1}{2}\left(c_{1}^{\prime}+c_{1}\right)$

$g_{12}=\frac{1}{2}\left(c_{1}^{\prime}-c_{1}\right)$

$g_{21}=\frac{1}{2}\left(c_{2}^{\prime}-c_{2}\right)$

$g_{22}=\frac{1}{2}\left(c_{2}^{\prime}+c_{2}\right)$.

In Eq. (A.5),

$$
\begin{aligned}
c_{1}= & \frac{1}{2 a^{2} \Delta_{1}}\left(a^{2}-2 a \beta_{11}+3 a \beta_{12}+a \beta_{22}+2 \beta_{12} \beta_{21}\right. \\
& \left.-2 \beta_{11} \beta_{22}\right),
\end{aligned}
$$

$$
\begin{aligned}
c_{2}= & \frac{1}{2 a^{2} \Delta_{1}}\left(a^{2}-2 a \beta_{22}+3 a \beta_{21}+a \beta_{11}+2 \beta_{12} \beta_{21}\right. \\
& \left.-2 \beta_{11} \beta_{22}\right), \\
c_{1}^{\prime}= & c_{1}-3 \frac{\beta_{12}}{a \Delta_{1}},
\end{aligned}
$$

$c_{2}^{\prime}=c_{2}-3 \frac{\beta_{21}}{a \Delta_{1}}$,

where

$\Delta_{1}=\frac{1}{a^{2}}\left(a^{2}+a \beta_{11}+a \beta_{22}-\beta_{12} \beta_{21}+\beta_{11} \beta_{22}\right)$,

and the relaxation coefficients $\beta_{11}, \beta_{12}, \beta_{21}$, and $\beta_{22}$ were defined by Eq. (11). In the limit of Eq. (6) or zero relaxation coefficients, Eqs. (A.5) and (A.6) reduce to $c_{1}=c_{2}=c_{1}^{\prime}=$ $c_{2}^{\prime}=g_{11}=g_{22}=1 / 2$ and $g_{12}=g_{21}=0$. In the other limit of very large relaxation coefficients, these equations become $c_{1}=c_{2}=c_{1}^{\prime}=c_{2}^{\prime}=g_{11}=g_{22}=-1$ and $g_{12}=g_{21}=0$.

The solution for the first reflected fields from the particle is

$\mu_{m \mathrm{p}}^{(1)}=a^{3} r^{-2} \cos \theta \sum_{i=1}^{2} g_{m i}\left|\nabla \mu_{i \infty}\right|$

$\mathbf{v}_{\mathrm{p}}^{(1)}=\frac{1}{2} U_{0} a^{3} r^{-3}\left(2 \cos \theta \mathbf{e}_{r}+\sin \theta \mathbf{e}_{\theta}\right)$.

The velocity distribution shown in Eq. (A.8b) is identical to the irrotational flow surrounding a rigid sphere moving with velocity $U_{0} \mathbf{e}_{z}$.

The boundary conditions for the $i$ th reflected fields from the wall are derived from Eqs. (12), (13), (25), and (26),

$$
\begin{aligned}
z=-b: & \mu_{m \mathrm{w}}^{(i)}=-\mu_{m \mathrm{p}}^{(i)} \\
& \mathbf{v}_{\mathrm{w}}^{(i)}=-\mathbf{v}_{\mathrm{p}}^{(i)}
\end{aligned}
$$




$$
\begin{array}{ll}
r \rightarrow \infty, z>-b: & \mu_{m \mathrm{w}}^{(i)} \rightarrow 0, \\
& \mathbf{v}_{\mathrm{w}}^{(i)} \rightarrow 0 .
\end{array}
$$

The solution of $\mu_{m \mathrm{w}}^{(1)}$ is obtained by applying Hankel transforms on variable $\rho$ in Eqs. (8) and (A.9a and A.9c) (taking $i=1$ ), with the result

$\mu_{m \mathrm{w}}^{(1)}=a^{3}(2 b+z)\left[(2 b+z)^{2}+\rho^{2}\right]^{-3 / 2} \sum_{i=1}^{2} g_{m i}\left|\nabla \mu_{i \infty}\right|$.

This reflected electrochemical potential field may be interpreted as arising from the reflection of the imposed field from a fictitious particle identical to the actual particle, its location being at the mirror-image position of the actual particle with respect to the plane $z=-b$ (i.e., at $\rho=0, z=-2 b$ ). The solution of $\mathbf{v}_{\mathrm{w}}^{(1)}$ can also be obtained by applying Hankel transforms to the Stokes equation (21) twice and to the boundary conditions (A.9b and A.9d), which results in

$$
\begin{aligned}
\mathbf{v}_{\mathrm{w}}^{(1)}= & -\frac{1}{2} U_{0} a^{3} \int_{0}^{\infty} \omega^{2}\left[E(\omega, z) J_{1}(\omega \rho) \mathbf{e}_{\rho}\right. \\
& \left.+F(\omega, z) J_{0}(\omega \rho) \mathbf{e}_{z}\right] d \omega,
\end{aligned}
$$

where

$E(\omega, z)=[2(b+z) \omega-1] \mathrm{e}^{-\omega(z+2 b)}$,

$F(\omega, z)=[2(b+z) \omega+1] \mathrm{e}^{-\omega(z+2 b)}$.

The contributions of $\mu_{m \mathrm{w}}^{(1)}$ and $\mathbf{v}_{\mathrm{w}}^{(1)}$ to the particle velocity are determined using Eq. (A.3),

$\mathbf{U}_{\mathrm{s}}^{(1)}=\sum_{m=1}^{2} G_{m}\left[\nabla \mu_{m \mathrm{w}}^{(1)}\right]_{r=0}=-\frac{1}{4} G \lambda^{3} U_{0} \mathbf{e}_{z}$,

$\mathbf{U}_{\mathrm{h}}^{(1)}=\left[\mathbf{v}_{\mathrm{w}}^{(1)}+\frac{a^{2}}{6} \nabla^{2} \mathbf{v}_{\mathrm{w}}^{(1)}\right]_{r=0}=-\frac{1}{4}\left(2 \lambda^{3}-\lambda^{5}\right) U_{0} \mathbf{e}_{z}$,

$\mathbf{U}^{(1)}=\mathbf{U}_{\mathrm{s}}^{(1)}+\mathbf{U}_{\mathrm{h}}^{(1)}=\frac{1}{4}\left[-(2+G) \lambda^{3}+\lambda^{5}\right] U_{0} \mathbf{e}_{z}$,

where

$G=\frac{1}{U_{0}} \sum_{m=1}^{2} \sum_{i=1}^{2} G_{m} g_{m i}\left|\nabla \mu_{i \infty}\right|$.

After the substitution of Eq. (14) for $\mu_{i \infty}$ and Eq. (A.5) for $g_{m i}$, Eq. (A.14) becomes

$G=\frac{k T\left|\nabla n^{\infty}\right|}{U_{0} n^{\infty}(\mathbf{0})} \sum_{m=1}^{2} G_{m} b_{m}$

where

$b_{m}=c_{m}^{\prime}-(-1)^{m} c_{m} \alpha$.

Equation (A.13a) shows that the reflected electrochemical potential field from the plane wall can decrease (if $G>0$ ) or increase (if $G<0$ ) the diffusiophoretic velocity of the particle from its undisturbed value, while Eq. (A.13b) indicates that the reflected velocity field is to decrease this velocity; the net effect of the reflected fields is expressed by Eq. (A.13c), which can enhance or retard the movement of the particle, depending on the combination of the values of $G$ and $\lambda$. When $G=0$, the reflected electrochemical potential field makes no contribution to the diffusiophoretic velocity. Eq. (A.13c) indicates that the wall correction to the velocity of the diffusiophoretic particle is $O\left(\lambda^{3}\right)$, which is weaker than that obtained for the corresponding sedimentation problem, in which the leading boundary effect is $O(\lambda)$. Note that the necessary condition for the wall enhancement on the diffusiophoretic motion to occur is a small value of $G$ and/or a value of $\lambda$ close to unity such that the relation $\lambda^{5}>(2+G) \lambda^{3}$ is warranted.

The solution for the second reflected fields from the particle is

$$
\begin{aligned}
\mu_{m \mathrm{p}}^{(2)}= & -\frac{1}{4} \lambda^{3} a^{3} r^{-2} \cos \theta \sum_{i=1}^{2} \sum_{j=1}^{2} g_{m j} g_{j i}\left|\nabla \mu_{i \infty}\right| \\
& +\frac{3}{16} \lambda^{4} a^{4} r^{-3}\left(3 \cos ^{2} \theta-1\right) \sum_{i=1}^{2} \sum_{j=1}^{2} h_{m j} g_{j i}\left|\nabla \mu_{i \infty}\right| \\
& +O\left(\lambda^{5} a^{5}\right),
\end{aligned}
$$

$$
\begin{aligned}
\mathbf{v}_{\mathrm{p}}^{(2)}= & U_{0}\left\{-\frac{1}{8} G \lambda^{3} a^{3} r^{-3}\left(2 \cos \theta \mathbf{e}_{r}+\sin \theta \mathbf{e}_{\theta}\right)\right. \\
& -\frac{9}{64}(5-4 H) \lambda^{4} a^{2} r^{-2}\left(3 \cos ^{2} \theta-1\right) \mathbf{e}_{r} \\
& -\frac{9}{16} H \lambda^{4} a^{4} r^{-4}\left[\left(3 \cos ^{2} \theta-1\right) \mathbf{e}_{r}+2 \sin \theta \cos \theta \mathbf{e}_{\theta}\right] \\
& +\frac{9}{64} \lambda^{4} a^{4} r^{-4}\left[\left(9 \cos ^{2} \theta-4\right) \mathbf{e}_{r}+54 \sin \theta \cos \theta \mathbf{e}_{\theta}\right] \\
& \left.+O\left(\lambda^{6} a^{4}\right)\right\},
\end{aligned}
$$

where

$H=\frac{1}{U_{0}} \sum_{m=1}^{2} \sum_{i=1}^{2} H_{m} g_{m i}\left|\nabla \mu_{i \infty}\right|$

with

$$
\begin{aligned}
H_{1}= & -\frac{\varepsilon k T}{\eta(Z e)^{2}}\left[\left(1+2 h_{11}-2 h_{21}\right) \bar{\zeta}\right. \\
& \left.+\left(1+2 h_{11}+2 h_{21}\right) \ln \cosh \bar{\zeta}\right], \\
H_{2}= & -\frac{\varepsilon k T}{\eta(Z e)^{2}}\left[\left(-1+2 h_{12}-2 h_{22}\right) \bar{\zeta}\right. \\
& \left.+\left(1+2 h_{12}+2 h_{22}\right) \ln \cosh \bar{\zeta}\right] .
\end{aligned}
$$

In Eqs. (A.17a) and (A.19),

$$
\begin{aligned}
& h_{11}=\frac{1}{2}\left(d_{1}^{\prime}+d_{1}\right), \\
& h_{12}=\frac{1}{2}\left(d_{1}^{\prime}-d_{1}\right), \\
& h_{21}=\frac{1}{2}\left(d_{2}^{\prime}-d_{2}\right),
\end{aligned}
$$


$h_{22}=\frac{1}{2}\left(d_{2}^{\prime}+d_{2}\right)$,

where

$$
\begin{aligned}
d_{1}= & \frac{1}{3 a^{2} \Delta_{2}}\left(a^{2}-3 a \beta_{11}+5 a \beta_{12}+2 a \beta_{22}+6 \beta_{12} \beta_{21}\right. \\
& \left.-6 \beta_{11} \beta_{22}\right) \\
d_{2}= & \frac{1}{3 a^{2} \Delta_{2}}\left(a^{2}-3 a \beta_{22}+5 a \beta_{21}+2 a \beta_{11}+6 \beta_{12} \beta_{21}\right. \\
& \left.-6 \beta_{11} \beta_{22}\right) \\
d_{1}^{\prime}= & d_{1}-\frac{10 \beta_{12}}{3 a \Delta_{2}} \\
d_{2}^{\prime}= & d_{2}-\frac{10 \beta_{21}}{3 a \Delta_{2}}
\end{aligned}
$$

and

$$
\Delta_{2}=\frac{1}{a^{2}}\left(a^{2}+2 a \beta_{11}+2 a \beta_{22}-4 \beta_{12} \beta_{21}+4 \beta_{11} \beta_{22}\right) .
$$

The boundary conditions for the second reflected fields from the wall are obtained by substituting the result of $\mu_{m p}^{(2)}$ and $\mathbf{v}_{\mathrm{p}}^{(2)}$ into Eq. (A.9), with which Eqs. (8) and (21) can be solved as before to yield

$$
\begin{aligned}
{\left[\nabla \mu_{m \mathrm{w}}^{(2)}\right]_{r=0}=} & \frac{1}{256}\left[16 \lambda^{6} \sum_{i=1}^{2} \sum_{j=1}^{2} g_{m j} g_{j i}\left|\nabla \mu_{i \infty}\right|\right. \\
+ & \left.18 \lambda^{8} \sum_{i=1}^{2} \sum_{j=1}^{2} h_{m j} g_{j i}\left|\nabla \mu_{i \infty}\right|+O\left(\lambda^{9}\right)\right] \mathbf{e}_{z}, \\
{\left[\mathbf{v}_{\mathrm{w}}^{(2)}+\frac{a^{2}}{6} \nabla^{2} \mathbf{v}_{\mathrm{w}}^{(2)}\right]_{r=0}=} & U_{0}\left\{\frac{1}{256}[32 G-27(5-4 H)] \lambda^{6}\right. \\
& +\frac{1}{512}[27(5-4 H)-32 G-180 H \\
& \left.-99] \lambda^{8}+O\left(\lambda^{9}\right)\right\} \mathbf{e}_{z} .
\end{aligned}
$$

The contribution of the second reflected fields to the particle velocity is obtained by combining Eqs. (A.3) and (A.23), which gives

$$
\begin{aligned}
\mathbf{U}^{(2)}= & U_{0}\left\{\frac{1}{256}\left[-27(5-4 H)+16\left(2 G+G^{\prime}\right)\right] \lambda^{6}\right. \\
& \left.+\frac{1}{128}\left(9-72 H+9 H^{\prime}-8 G\right) \lambda^{8}+O\left(\lambda^{9}\right)\right\} \mathbf{e}_{z},
\end{aligned}
$$

where

$$
\begin{aligned}
G^{\prime} & =\frac{1}{U_{0}} \sum_{m=1}^{2} \sum_{i=1}^{2} \sum_{j=1}^{2} G_{m} g_{m j} g_{j i}\left|\nabla \mu_{i \infty}\right| \\
H^{\prime} & =\frac{1}{U_{0}} \sum_{m=1}^{2} \sum_{i=1}^{2} \sum_{j=1}^{2} G_{m} h_{m j} g_{j i}\left|\nabla \mu_{i \infty}\right| .
\end{aligned}
$$

After the substitution of Eq. (14) for $\mu_{i \infty}$ and Eq. (A.5) for $g_{m i}$, Eqs. (A.18) and (A.25) become

$$
\begin{aligned}
& H=\frac{k T\left|\nabla n^{\infty}\right|}{U_{0} n^{\infty}(\mathbf{0})} \sum_{m=1}^{2} H_{m} b_{m}, \\
& G^{\prime}=\frac{k T\left|\nabla n^{\infty}\right|}{U_{0} n^{\infty}(\mathbf{0})} \sum_{m=1}^{2} \sum_{j=1}^{2} G_{m} g_{m j} b_{j}, \\
& H^{\prime}=\frac{k T\left|\nabla n^{\infty}\right|}{U_{0} n^{\infty}(\mathbf{0})} \sum_{m=1}^{2} \sum_{j=1}^{2} G_{m} h_{m j} b_{j} .
\end{aligned}
$$

Evidently, $\mathbf{U}^{(3)}$ will be $O\left(\lambda^{9}\right)$. With the substitution of Eqs. (A.13c) and (A.24) into Eq. (A.2), the particle velocity can be expressed as $\mathbf{U}=U \mathbf{e}_{z}$ with

$$
\begin{aligned}
U= & U_{0}\left\{1-\frac{1}{4}(2+G) \lambda^{3}+\frac{1}{4} \lambda^{5}\right. \\
& -\frac{1}{256}\left[27(5-4 H)-16\left(2 G+G^{\prime}\right)\right] \lambda^{6} \\
& \left.+\frac{1}{128}\left(9-72 H+9 H^{\prime}-8 G\right) \lambda^{8}+O\left(\lambda^{9}\right)\right\} .
\end{aligned}
$$

The particle migrates along the imposed electrolyte concentration gradient at a rate that can increase or decrease as the particle approaches the wall. Owing to the linearity of the problem, the above analysis is valid when the particle is either approaching the plane wall or receding from it.

For the problem of electrophoretic motion of a spherical particle caused by a constant external electric field $\mathbf{E}^{\infty}=E^{\infty} \mathbf{e}_{z}$ perpendicular to a conducting plane, the above analysis still applies, but now $U_{0}$ is the electrophoretic velocity of an isolated sphere given by Eq. (5) and the expressions for $G, H, G^{\prime}$, and $H^{\prime}$ given by Eqs. (A.15), (A.26), and (A.27) become

$$
\begin{aligned}
& G=-\frac{Z e E^{\infty}}{U_{0}} \sum_{m=1}^{2}(-1)^{m} G_{m} c_{m}, \\
& H=-\frac{Z e E^{\infty}}{U_{0}} \sum_{m=1}^{2}(-1)^{m} H_{m} c_{m}, \\
& G^{\prime}=-\frac{Z e E^{\infty}}{U_{0}} \sum_{m=1}^{2} \sum_{j=1}^{2}(-1)^{j} G_{m} g_{m j} c_{j}, \\
& H^{\prime}=-\frac{Z e E^{\infty}}{U_{0}} \sum_{m=1}^{2} \sum_{j=1}^{2}(-1)^{j} G_{m} h_{m j} c_{j} .
\end{aligned}
$$

Using the same method of reflections, Keh and Anderson [18] obtained an asymptotic solution for the electrophoretic mobility of a dielectric sphere surrounded by an infinitesimally thin electric double layer normal to a conducting plane wall correct to $O\left(\lambda^{6}\right)$. In the limit of Eq. (6), the formula (A.28) with coefficients given by Eqs. (A.29) and (A.30) is consistent with this earlier solution. 


\section{A.2. Motion normal to two parallel plane walls}

For the problem of diffusiophoretic or electrophoretic motion of a spherical particle perpendicular to two infinite plane walls with equal distances from the particle, the boundary conditions corresponding to governing equations (8) and (21) are given by Eqs. (10), (12), (13), and (24)-(26) with $c=b$. With $\lambda=a / b \ll 1$, the series expansions of the electrochemical potentials of the ions, fluid velocity, and particle velocity given by Eqs. (A.1), (A.2), and (A.8) remain valid here. From Eqs. (12), (13), (25), and (26), the boundary conditions for $\mu_{m \mathrm{w}}^{(i)}$ and $\mathbf{v}_{\mathrm{w}}^{(i)}$ are found to be

$$
\begin{array}{r}
|z|=b: \quad \mu_{m \mathrm{w}}^{(1)}=-\mu_{m \mathrm{p}}^{(1)}, \\
\mathbf{v}_{\mathrm{w}}^{(1)}=-\mathbf{v}_{\mathrm{p}}^{(1)} ; \\
r \rightarrow \infty,|z| \leqslant b: \quad \mu_{m \mathrm{w}}^{(1)} \rightarrow 0, \\
\quad \mathbf{v}_{\mathrm{w}}^{(1)} \rightarrow 0 .
\end{array}
$$

The first wall-reflected fields can be solved by the same method as used for the case of a single plane wall in the previous subsection, with the results

$$
\begin{aligned}
\mu_{m \mathrm{w}}^{(1)}= & -a \lambda^{2} \int_{0}^{\infty} \frac{1+e^{-2 t}}{\sinh (2 t)} \sinh \left(\frac{t}{b} z\right) t J_{0}\left(\frac{t}{b} \rho\right) d t \\
& \times \sum_{i=1}^{2} g_{m i}\left|\nabla \mu_{i \infty}\right|, \\
\mathbf{v}_{\mathrm{w}}^{(1)}= & -\frac{1}{2} U_{0} \lambda^{3} \int_{0}^{\infty} t^{2}\left[E(t, z) J_{1}\left(\frac{t}{b} \rho\right) \mathbf{e}_{\rho}\right. \\
& \left.+F(t, z) J_{0}\left(\frac{t}{b} \rho\right) \mathbf{e}_{z}\right] d t
\end{aligned}
$$

where

$$
\begin{aligned}
E(\alpha, z)= & \frac{2}{2 \alpha+\sinh (2 \alpha)} \\
& \times\left[\left(1-\alpha-\mathrm{e}^{-\alpha} \sinh \alpha\right) \sinh \left(\frac{\alpha}{b} z\right)\right. \\
& \left.+\frac{\alpha}{b} z \cosh \left(\frac{\alpha}{b} z\right)\right], \\
F(\alpha, z)= & \frac{2}{2 \alpha+\sinh (2 \alpha)} \\
& \times\left[\left(\alpha+\mathrm{e}^{-\alpha} \sinh \alpha\right) \cosh \left(\frac{\alpha}{b} z\right)\right. \\
& \left.-\frac{\alpha}{b} z \sinh \left(\frac{\alpha}{b} z\right)\right],
\end{aligned}
$$

$t=\omega b$, and $U_{0}$ is given by Eqs. (7) and (5) for the cases of diffusiophoresis and electrophoresis, respectively. The contributions of $\mu_{m \mathrm{w}}^{(1)}$ and $\mathbf{v}_{\mathrm{w}}^{(1)}$ to the particle velocity are determined using Eq. (A.3), which lead to a result similar to Eq. (A.13),

$\mathbf{U}_{\mathrm{s}}^{(1)}=-\bar{d}_{1} G \lambda^{3} U_{0} \mathbf{e}_{z}$,

$$
\begin{aligned}
& \mathbf{U}_{\mathrm{h}}^{(1)}=\left[-\bar{d}_{2} \lambda^{3}+\bar{d}_{3} \lambda^{5}\right] U_{0} \mathbf{e}_{z}, \\
& \mathbf{U}^{(1)}=\mathbf{U}_{\mathrm{s}}^{(1)}+\mathbf{U}_{\mathrm{h}}^{(1)}=\left[-\left(\bar{d}_{2}+\bar{d}_{1} G\right) \lambda^{3}+\bar{d}_{3} \lambda^{5}\right] U_{0} \mathbf{e}_{z},
\end{aligned}
$$

where $G$ is given by Eq. (A.14) (or by Eqs. (A.15) and (A.29a) for the relevant cases), and

$$
\begin{aligned}
& \bar{d}_{1}=\int_{0}^{\infty} \frac{1+\mathrm{e}^{-2 t}}{\sinh (2 t)} t^{2} d t=0.60103, \\
& \bar{d}_{2}=\int_{0}^{\infty} \frac{\mathrm{t}+\mathrm{e}^{-t} \sinh t}{2 t+\sinh (2 t)} t^{2} d \alpha=0.79076, \\
& \bar{d}_{3}=\frac{1}{3} \int_{0}^{\infty} \frac{t^{4}}{2 t+\sinh (2 t)} d t=0.44175 .
\end{aligned}
$$

Again, Eq. (A.34a) shows that the reflected electrochemical potential field from the confining walls can decrease (if $G>0$ ) or increase (if $G<0$ ) the particle velocity, while Eq. (A.34b) indicates that the reflected velocity field is to decrease this velocity; the net effect is expressed by Eq. (A.34c), which can enhance or retard the movement of the particle, depending on the combination of the values of $G$ and $\lambda$. Equation (A.34c) indicates that the necessary condition for the wall enhancement on the diffusiophoretic or electrophoretic motion to occur is a small value of $G$ and a value of $\lambda$ close to unity such that the relation $\bar{d}_{3} \lambda^{5}>\left(\bar{d}_{2}+\bar{d}_{1} G\right) \lambda^{3}$ is warranted.

Analogous to the previous case, the results of the second reflections can be obtained as

$\mu_{m \mathrm{p}}^{(2)}=-\bar{d}_{1} \lambda^{3} a^{3} r^{-2} \cos \theta \sum_{i=1}^{2} \sum_{j=1}^{2} g_{m j} g_{j i}\left|\nabla \mu_{i \infty}\right|+O\left(\lambda^{5} a^{5}\right)$

$\mathbf{v}_{\mathrm{p}}^{(2)}=-\frac{1}{2} U_{0} \bar{d}_{1} G \lambda^{3} a^{3} r^{-3}\left(2 \cos \theta \mathbf{e}_{r}+\sin \theta \mathbf{e}_{\theta}\right)+O\left(\lambda^{6} a^{4}\right)$,

$\left[\nabla \mu_{m \mathrm{w}}^{(2)}\right]_{r=0}=\left[\bar{d}_{1}^{2} \lambda^{6} \sum_{i=1}^{2} \sum_{j=1}^{2} g_{m j} g_{j i}\left|\nabla \mu_{i \infty}\right|+O\left(\lambda^{9}\right)\right] \mathbf{e}_{z}$,

(A.36c)

$\left[\mathbf{v}_{\mathrm{w}}^{(2)}+\frac{a^{2}}{6} \nabla^{2} \mathbf{v}_{\mathrm{w}}^{(2)}\right]_{r=0}$

$$
=U_{0}\left[\bar{d}_{1} \bar{d}_{2} G \lambda^{6}-\bar{d}_{1} \bar{d}_{3} G \lambda^{8}+O\left(\lambda^{9}\right)\right] \mathbf{e}_{z},
$$

and

$\mathbf{U}^{(2)}=\left[\left(\bar{d}_{1}^{2} G^{\prime}+\bar{d}_{1} \bar{d}_{2} G\right) \lambda^{6}-\bar{d}_{1} \bar{d}_{3} G \lambda^{8}+O\left(\lambda^{9}\right)\right] U_{0} \mathbf{e}_{z}$.

Note that the $\lambda^{5} a^{5}$ and $\lambda^{6} a^{4}$ terms in the expressions for $\mu_{m p}^{(2)}$ and $\mathbf{v}_{\mathrm{p}}^{(2)}$ vanish.

With the combination of Eqs. (A.2), (A.34c), and (A.37), the particle velocity can be expressed as $\mathbf{U}=U \mathbf{e}_{z}$ with

$$
\begin{aligned}
U= & U_{0}\left[1-\left(\bar{d}_{2}+\bar{d}_{1} G\right) \lambda^{3}+\bar{d}_{3} \lambda^{5}\right. \\
& \left.+\left(\bar{d}_{1}^{2} G^{\prime}+\bar{d}_{1} \bar{d}_{2} G\right) \lambda^{6}-\bar{d}_{1} \bar{d}_{3} G \lambda^{8}+O\left(\lambda^{9}\right)\right] .
\end{aligned}
$$


This result is valid for a particle undergoing diffusiophoresis or electrophoresis toward either of the two plane walls.

As discussed in Sections 3 and 4, the boundary effects on diffusiophoretic and electrophoretic motions in general are quite complicated and no simple rule is able to make an adequate prediction for such complicated phenomena. Thus, limited numerical solutions with interpolation and extrapolation are awkward to be used in practical applications. Therefore, the closedform analytical results obtained in this appendix, which can be conveniently used in the calculations for various cases with $\kappa a>20$ (without the need of a computer), should be a favorable contribution to the evaluation and understanding of the boundary effects on diffusiophoresis and electrophoresis.

\section{Appendix B. Definitions of some functions in Section 2}

The functions $\delta_{n}^{(i)}$ with $i=1,2,3$, and 4 in Eqs. (18), (20), (33), and (38) are defined by

$$
\begin{aligned}
& \delta_{n}^{(1)}(r, \theta)=\int_{0}^{\infty} \omega(\sinh \tau)^{-1}\left[-B_{1 n}^{\prime \prime}(\omega,-b) \sinh \xi\right. \\
& \left.+B_{1 n}^{\prime \prime}(\omega, c) \sinh \sigma\right] J_{0}(\omega r \sin \theta) d \omega \\
& +r^{-n-1} P_{n}(\cos \theta) \text {, } \\
& \delta_{n}^{(2)}(r, \theta)=\int_{0}^{\infty} \omega^{2}(\sinh \tau)^{-1}\left\{\operatorname { s i n } \theta \left[B_{1 n}^{\prime \prime}(\omega,-b) \sinh \xi\right.\right. \\
& \left.-B_{1 n}^{\prime \prime}(\omega, c) \sinh \sigma\right] J_{1}(\omega r \sin \theta) \\
& +\cos \theta\left[-B_{1 n}^{\prime \prime}(\omega,-b) \cosh \xi\right. \\
& \left.\left.+B_{1 n}^{\prime \prime}(\omega, c) \cosh \sigma\right] J_{0}(\omega r \sin \theta)\right\} d \omega \\
& -(n+1) r^{-n-2} P_{n}(\cos \theta) \text {, } \\
& \delta_{n}^{(3)}(r, \theta)=\int_{0}^{\infty} \omega^{2}(\sinh \tau)^{-1}\left\{\operatorname { c o s } \theta \left[B_{1 n}^{\prime \prime}(\omega,-b) \sinh \xi\right.\right. \\
& \left.-B_{1 n}^{\prime \prime}(\omega, c) \sinh \sigma\right] J_{1}(\omega r \sin \theta) \\
& +\sin \theta\left[B_{1 n}^{\prime \prime}(\omega,-b) \cosh \xi\right. \\
& \text { - } \left.\left.B_{1 n}^{\prime \prime}(\omega, c) \cosh \sigma\right] J_{0}(\omega r \sin \theta)\right\} d \omega \\
& +n r^{-n-2}\left[P_{n}(\cos \theta) \cos \theta\right. \\
& \left.-P_{n-1}(\cos \theta)\right] \csc \theta \text {, } \\
& \delta_{n}^{(4)}(r, \theta)=\int_{0}^{\infty} \omega^{3}(\sinh \tau)^{-1}\left\{\operatorname { s i n } 2 \theta \left[B_{1 n}^{\prime \prime}(\omega,-b) \cosh \xi\right.\right. \\
& \left.-B_{1 n}^{\prime \prime}(\omega, c) \cosh \sigma\right] J_{1}(\omega r \sin \theta) \\
& +\cos ^{2} \theta\left[-B_{1 n}^{\prime \prime}(\omega,-b) \sinh \xi\right. \\
& \left.+B_{1 n}^{\prime \prime}(\omega, c) \sinh \sigma\right] J_{0}(\omega r \sin \theta) \\
& +\sin ^{2} \theta\left[-B_{1 n}^{\prime \prime}(\omega,-b) \sinh \xi\right. \\
& \left.+B_{1 n}^{\prime \prime}(\omega, c) \sinh \sigma\right]\left[J_{2}(\omega r \sin \theta)\right. \\
& \left.\left.-J_{0}(\omega r \sin \theta)\right] / 2\right\} d \omega \\
& +(n+1)(n+2) r^{-n-3} P_{n}(\cos \theta) \text {, }
\end{aligned}
$$

and the functions $\gamma_{i n}^{(j)}$ for $i$ and $j$ equal to 1 or 2 in Eqs. (31) and (32) are defined by

$$
\begin{aligned}
\gamma_{i n}^{(1)}(r, \theta)= & -\int_{0}^{\infty}\left[G_{+}^{\prime \prime}(\sigma, \xi) B_{i n}^{\prime}(\omega,-b)-G_{+}^{\prime \prime}(\xi, \sigma) B_{i n}^{\prime}(\omega, c)\right. \\
& -G_{+}^{\prime}(\sigma, \xi) B_{i n}^{\prime \prime}(\omega,-b) \\
& \left.+G_{+}^{\prime}(\xi, \sigma) B_{i n}^{\prime \prime}(\omega, c)\right] \omega J_{1}(\omega r \sin \theta) d \omega \\
& -r^{-n+2 i-3}\left[(n+1) G_{n+1}^{-1 / 2}(\cos \theta) \csc \theta\right. \\
& \left.-2(i-1) G_{n}^{-1 / 2}(\cos \theta) \cot \theta\right],
\end{aligned}
$$

$$
\begin{aligned}
\gamma_{i n}^{(2)}(r, \theta)= & -\int_{0}^{\infty}\left[-G_{-}^{\prime}(\sigma, \xi) B_{i n}^{\prime}(\omega,-b)+G_{-}^{\prime}(\xi, \sigma) B_{i n}^{\prime}(\omega, c)\right. \\
& +G_{-}^{\prime \prime}(\sigma, \xi) B_{i n}^{\prime \prime}(\omega,-b) \\
& \left.-G_{-}^{\prime \prime}(\xi, \sigma) B_{i n}^{\prime \prime}(\omega, c)\right] \omega J_{0}(\omega r \sin \theta) d \omega \\
& -r^{-n+2 i-3}\left[P_{n}(\cos \theta)+2(i-1) G_{n}^{-1 / 2}(\cos \theta)\right],
\end{aligned}
$$

where

$$
\begin{aligned}
& B_{1 n}^{\prime}(\omega, z)=-\frac{1}{n !}\left(\frac{\omega|z|}{z}\right)^{n-1} \mathrm{e}^{-\omega|z|}, \\
& B_{1 n}^{\prime \prime}(\omega, z)=-\frac{\omega^{n-1}}{n !}\left(\frac{|z|}{z}\right)^{n} \mathrm{e}^{-\omega|z|}, \\
& B_{2 n}^{\prime}(\omega, z)=-\frac{1}{n !}\left(\frac{\omega|z|}{z}\right)^{n-3}[(2 n-3) \omega|z|-n(n-2)] \mathrm{e}^{-\omega|z|}, \\
& B_{2 n}^{\prime \prime}(\omega, z)=-\frac{\omega^{n-3}}{n !}\left(\frac{|z|}{z}\right)^{n}[(2 n-3) \omega|z| \\
& G_{ \pm}^{\prime}(\mu, v)=\tau^{*} \mu \nu\left(\mu^{\prime} \pm \tau^{\prime} v^{\prime}\right), \\
& G_{ \pm}^{\prime \prime}(\mu, v)=\tau^{*}\left[v\left(\cosh \mu-\tau^{\prime} v^{\prime}\right) \pm \mu\left(\mu^{\prime}-\tau^{\prime} \cosh v\right)\right], \\
& \mu^{\prime}=\frac{\sinh \mu}{\mu}, \\
& \tau^{\prime}=\frac{\sinh \tau}{\tau}, \\
& \sigma=\omega(r \cos \theta+b), \\
& \tau=\omega(b+c) . \\
& \tau^{*}=\frac{\sinh ^{\prime} v}{\sinh ^{2} \tau-\tau^{2}}, \\
& \xi=\omega(r \cos \theta-c),
\end{aligned}
$$

\section{References}

[1] D.C. Henry, Proc. R. Soc. London A 133 (1931) 106.

[2] F.A. Morrison, J. Colloid Interface Sci. 34 (1970) 210.

[3] S.S. Dukhin, B.V. Derjaguin, in: E. Matijevic (Ed.), Surface and Colloid Science, vol. 7, Wiley, New York, 1974.

[4] J.H. Masliyah, Electrokinetic Transport Phenomena, AOSTRA, Edmonton, 1994.

[5] D.C. Prieve, Adv. Colloid Interface Sci. 16 (1982) 321.

[6] D.C. Prieve, J.L. Anderson, J.P. Ebel, M.E. Lowell, J. Fluid Mech. 148 (1984) 247.

[7] J.L. Anderson, Annu. Rev. Fluid Mech. 21 (1989) 61. 
[8] R.W. O’Brien, L.R. White, J. Chem. Soc. Faraday. Trans. 2 (74) (1978) 1607.

[9] R.W. O’Brien, J. Colloid Interface Sci. 92 (1983) 204.

[10] S.B. Chen, H.J. Keh, J. Fluid Mech. 238 (1992) 251.

[11] D.C. Prieve, R. Roman, J. Chem. Soc. Faraday. Trans. 2 (83) (1987) 1287.

[12] H.J. Keh, S.B. Chen, Langmuir 9 (1993) 1142.

[13] Y. Pawar, Y.E. Solomentsev, J.L. Anderson, J. Colloid Interface Sci. 155 (1993) 488.

[14] H.A. Stone, S. Kim, AIChE J. 47 (2001) 1250.

[15] C. Ye, D. Li, Microfluid Nanofluid 1 (2004) 52.

[16] H. Liu, H.H. Bau, H.H. Hu, Langmuir 20 (2004) 2628.

[17] H.N. Unni, H.J. Keh, C. Yang, Electrophoresis 28 (2007) 658.
[18] H.J. Keh, J.L. Anderson, J. Fluid Mech. 153 (1985) 417.

[19] H.J. Keh, S.B. Chen, J. Fluid Mech. 194 (1988) 377.

[20] H.J. Keh, L.C. Lien, J. Fluid Mech. 224 (1991) 305.

[21] M. Loewenberg, R.H. Davis, J. Fluid. Mech. 288 (1995) 103.

[22] H.J. Keh, J.Y. Chiou, AIChE J. 42 (1996) 1397.

[23] E. Yariv, H. Brenner, J. Fluid Mech. 484 (2003) 85.

[24] T.H. Hsieh, H.J. Keh, J. Colloid Interface Sci. 315 (2007) 343.

[25] H.J. Keh, J.S. Jan, J. Colloid Interface Sci. 183 (1996) 458.

[26] P.Y. Chen, H.J. Keh, J. Colloid Interface Sci. 286 (2005) 774.

[27] P. Ganatos, S. Weinbaum, R. Pfeffer, J. Fluid Mech. 99 (1980) 739.

[28] J. Happel, H. Brenner, Low Reynolds Number Hydrodynamics, Nijhoff, The Netherlands, 1983.

[29] H.J. Tu, H.J. Keh, J. Colloid Interface Sci. 231 (2000) 265. 